\author{
Universidade de São Paulo \\ Instituto de Astronomia, Geofísica e Ciências Atmosféricas \\ Department: Geofísica IAG-USP \\ Program: Geofísica
}

Camilo Muñoz Lopez

Parameter optimization of automatic phase detection and picking algorithms - Application in São Paulo University Seismological Center and Colombian National Seismic Network.

São Paulo

2021 


\section{Camilo Muñoz Lopez}

\section{Parameter optimization of automatic phase detection and picking algorithms - Application in São Paulo University Seismological Center and Colombian National Seismic}

Network.

\section{Revised Version}

Versão original encontra-se disponível na unidade que aloja o Programa de Pós-graduação

MSc Thesis presented to the Graduate Program in Geofísica at the Instituto de Astronomia, Geofísica e Ciências Atmosféricas, Universidade de São Paulo, Brazil to obtain the degree of Master of Science.

Concentration area: Seismology Advisor: Prof. Dr. Marcelo Sousa de Assumpção

\section{São Paulo}




\begin{abstract}
The accurate and efficient analysis of seismic data requires the implementation of automatic processing algorithms. Therefore, the reliability and quality of these automatic results have become critical requirements for seismological networks. Two methodologies, Grid-search and Bayesian optimization, were used to optimize the automatic detection and phase picking parameters in SeisComP. These methodologies were tested using a set of stations selected from two seismological networks the Brazilian Seismic Network (RSBR) and Colombian National Seismological Network (RSNC). A comparison of manual and previous automatic locations, revealed numerous missing events and others with low-quality locations in automatic databases. We selected 508 manual events from 2017/01/01 to 2020/07/31 in Brazil, and 532 manual events from 2019/02/01 to 2019/02/15 near the Bucaramanga Nest in Colombia, as training data sets for the optimization process.

A code was implemented to use an iterative grid-search to optimize the picking parameters. In addition, the Optuna Python package was used to implement the Bayesian optimization. Selected events were used as a training set, and an iterative process according to the Bayesian method was used. The results of both methodologies were compared.

Both methodologies improved the system performance by increasing the number of picks and detections. Grid-search allowed us to perform a complete analysis of the results examining the entire space of parameters. However, Grid-search lose efficiency while increasing the number of parameters being optimized. On the other hand, the Bayesian algorithm is computationally more efficient by not exploring the entire parameter space.
\end{abstract}


After the optimization process, automatic picks associated with $\mathrm{P}$ phases increases by $78 \%$ (76 picks) and 56\% (903 picks) for RSBR and RSNC, respectively. Although not all new picks belong to new events, the number of locations calculated using new automatic picks doubled the automatic locations determined by the systems before the optimization process for both networks.

Seismological centers could implement methodologies such as Grid-search or Bayesian optimization to improve their automatic processing systems. Besides, the standardization of these methodologies would help to make their implementation easier. 


\section{Otimização de parâmetros dos algoritmos de detecção automática e picado}

de fases - Aplicação no Centro Sismológico da Universidade de São Paulo e na Rede Sismológica Nacional da Colômbia.

A análise precisa e eficiente de dados sísmicos requer a implementação de algoritmos de processamento automático. Portanto, a confiabilidade e a qualidade desses resultados automáticos tornaram-se requisitos críticos para redes sismológicas. Duas metodologias, Grid-Search e algoritmo Bayesiano, foram utilizadas para otimizar a detecção automática de eventos e os parâmetros de seleção de fases no software SeisComP. Essas metodologias foram testadas com um conjunto de estações selecionadas de duas redes sismológicas, Rede Sismográfica Brasileira (RSBR) e a Rede Sismológica Nacional da Colômbia (RSNC). Depois de comparar as localizações manuais e automáticas, encontramos vários eventos ausentes e outros com localizações de baixa qualidade nos bancos de dados automáticos. Selecionamos 508 eventos manuais no período 2017/01/01 - 2020/07/31 no Brasil, e 532 eventos manuais no período 2019/02/01 - 2019/02/15 próximos ao Ninho de Bucaramanga na Colômbia, como conjuntos de dados de treinamento para o processo de otimização.

Foi implementado um código para usar Grid-Search como uma metodologia de otimização; este código faz um processo iterativo que gera picks automáticos modificando os parâmetros. O pacote Optuna foi utilizado para implementar o algoritmo Bayesiano como metodologia de otimização. Os eventos selecionados foram usados como um conjunto de treinamento, e um processo iterativo de acordo com o método Bayesiano foi usado.

Ambas as metodologias melhoraram o desempenho do sistema, aumentando o número de picks e detecções. Grid-Search nos permitiu realizar uma análise completa dos resultados examinando todo o espaço de parâmetros. Grid-Search, no entanto, aumenta o tempo de computação ao testar muitos valores dos parâmetros envolvidos no processo de otimização. Por outro lado, o algoritmo Bayesiano pode ser implementado usando vários parâmetros sem aumentar o tempo de computação.

Após o processo de otimização, os picks automáticos aumentam em $78 \%$ e $56 \%$ para RSBR e RSNC, respectivamente. Embora nem todos os novos picks pertençam a novos eventos, O número de localizações calculadas com os novos pikcs automáticos, 
dobrou as localizações automáticas determinadas pelos sistemas antes do processo de otimização para ambas as redes.

Centros sismológicos podem implementar metodologias como Grid-Search ou algoritmo Bayesiano para melhorar seus sistemas de processamento automático. Além disso, a padronização dessas metodologias ajudaria a facilitar sua implementação. 


\section{Contents}

List of Figures

List of Tables Xii

1 Introduction 2

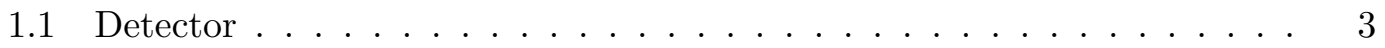

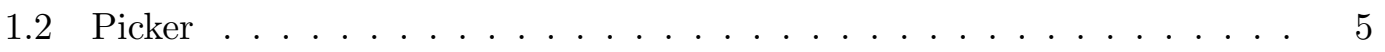

1.3 Parameter optimization: . . . . . . . . . . . . 7

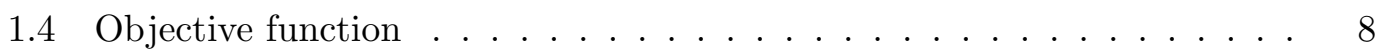

1.5 Grid Search . . . . . . . . . . . . . . . . . . . . . 9

1.6 Bayesian . . . . . . . . . . . . . . . . . . . 10 10

2 Paper

3 Additional Results 35

3.1 Grid-search(recall) results for second data set (Jan/2017 - June/2020) . 36

3.2 Bayesian(f1-score) results for second data set (Jan/2017 - June/2020) . 40

3.3 Grid-search(recall) results for first data set (Jan to July/2017) . . . . . 45

3.3.1 Good performance in real automatic picks . . . . . . . . . . 45

3.3 .2 Numerous false picks . . . . . . . . . . . . . . . . . 46

3.3 .3 Good performance on false picks . . . . . . . . . . 49

3.3 .4 Few manual picks . . . . . . . . . . . . . . . . 51

3.3.5 Few real automatic picks before optimization process . . . . . . 51

4 Conclusion

4.1 Final Conclusion . . . . . . . . . . . . . . . . . 5 53

4.2 Principal Achievements . . . . . . . . . . . . 54 
4.3 Future Work . . . . . . . . . . . . . . . . . . 54 


\section{List of Figures}

$1.1 \quad \mathrm{STA} / \mathrm{LTA}$ detector $\ldots \ldots \ldots \ldots \ldots \ldots$

1.2 AIC picker . . . . . . . . . . . . . . . . 6

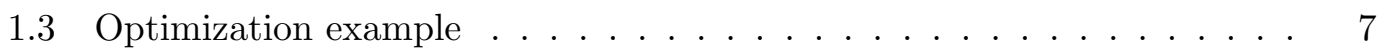

1.4 Precision and Recall . . . . . . . . . . . . . . . . . . . . 9

1.5 Grid-search and Bayesian . . . . . . . . . . . . . . . . 10

1.6 1D Gaussian process . . . . . . . . . . . . . . . . . . . . 11

1.7 1D Gaussian process iteration . . . . . . . . . . . . . . 13

3.1 Probability density functions for each parameter $\ldots \ldots \ldots 36$

3.2 False picks results for defect BW optimization . . . . . . . . . . . . 37

3.3 Probability density function for each parameter results . . . . . . . . . . 37

3.4 False and real picks results for each parameter optimization process in CNLB station. . . . . . . . . . . . . . . . . . 38

3.5 Bayesian and f1-score optimization results for SJMB station . . . . . . 4 40

3.6 SNR optimization results for AGLP station. . . . . . . . . . . . . 41

3.7 Bayesian and f1-score optimization results for BSCB station . . . . . . . 42

3.8 Objective value results for RCLB station . . . . . . . . . . . . . 43

3.9 Parameter optimization results for station DUB01 . . . . . . . 45

3.10 Parameter optimization results for station ALF01 . . . . . . . . . . 47

3.11 Parameter optimization results for station SJMB . . . . . . . . . . . 48

3.12 Parameter optimization results for station RIB01 . . . . . . . . . . . 4 49

3.13 Parameter optimization results for station SALV . . . . . . . . . 50 


\section{List of Tables}

3.1 Table with the results using Grid-search(recall) optimization for second data set (Jan/2017 - June/2020). BW: Butterworth. lf: Low frequency. hf: High frequency. man: Manual picks. auto: Automatic picks before optimization. real: Automatic picks after optimization. False: False automatic picks after optimization. . . . . . . . . . . . . 39

3.2 Table with the results using Bayesian(f1-score) optimization for second data set (Jan/2017 - June/2020). BW: Butterworth. lf: Low frequency. hf: High frequency. man: Manual picks. auto: Automatic picks before optimization. real: Automatic picks after optimization. False: False automatic picks after optimization. . . . . . . . . . . 44

3.3 Table with the results of the parameters optimized. BW: Butterworth. lf: low frequency. hf: high frequency. man: manual picks. auto: percentage of manual picks identified automatically before optimization. real: percentage of manual picks identified automatically after optimization. fake: false automatic picks after optimization. Blue color: stations with more than $75 \%$ of manual picks identified automatically after optimization. Red color: stations with poor results of false picks, stations with less than $5 \%$ of manual picks identified before optimization, and parameters identified which generate poor results of real or false picks. Pink color: stations with less than five manual picks. . . . . . . . . . . 52 


\section{Content design}

This document is divided into three principal sections:

- Introduction: Here, we present concepts related to algorithms and methods using during this research.

- Paper: This section contains the paper resulting from this study which includes abstract, introduction, data, methods, results, discussion, and conclusions.

- Additional results: In this section, we present results that were not included in the paper. Results from the two periods selected in this study are presented. 


\section{1}

\section{Introduction}

The increasing amount of seismic data recorded and stored by seismological networks makes manual processing difficult. Manual picking of seismic phases has been the methodology most used in seismological centers, but it is a time-consuming process and includes the error associated with the person's expertise in charge (Leonard, 2000). A prompt and good quality response is a priority for most seismological networks. Accurate and efficient analysis of seismic data requires the implementation of automatic processing algorithms. Therefore, the reliability and quality of the results produced automatically have become a critical requirement for the seismological networks.

To improve the automatic processing system results, we developed a new workflow to optimize parameters used by the detector and picker algorithms. The optimization process was done using two methodologies; grid-search and Bayesian. Both methods use a good quality dataset as training data and seek to reproduce this data after parameter optimization. The resulting parameters must improve the automatic results of the realtime data processing and, therefore, the reliability and quality of the results produced by the networks.

We used data from two seismological networks; the Brazilian Seismic Network (RSBR) (Bianchi et al., 2018) and Colombian National Seismological Network (RSNC) (Colombiano, 1993). The RSBR is the national network of Brazil and is operated by Sao Paulo university seismological center (USP-IAG), Rio Grande do Norte university seismological laboratory (UFRN), national observatory (ON) and Brasilia University observatory (UNB). This network has 93 stations installed with broadband sensors and real-time transmission to date. The RSNC is the national network of Colombia and is 
operated by the Colombian Geological Survey, this network has 65 stations installed with broadband sensors and some of them with short period co-located sensors, all of them use real-time transition.

The main objective of this study is to improve the quantity an quality of picks and therefore locations produced automatically by the optimization of critical detector and picker parameters.

The arrival picking of the seismic phases is the first step of automatic processing. The most accepted methodologies use variations in amplitude or frequency between the seismic noise and the earthquake signal (Withers et al., 1998) to automatically identify the seismic phases. In this article, we use the approach used by Allen (1982), separating the arrival picking into two sub-processes, the detection to find the seismic phases and the picker to estimate the onset time.

\subsection{Detector}

Detection seeks to identify arrivals of seismic phases without the need of accurately determining their onset times. Several algorithms have been proposed for the automatic detection of events (Allen, 1978) (Baer and Kradolfer, 1987). Most authors calculate a characteristic function $(C F)$ which is a substitute function calculated by applying to the original signal some operation to highlight the onset of $\mathrm{P}$ or $\mathrm{S}$ waves. The $C F$ can be calculated using band-pass filter, absolute amplitude, power, polarization indicator, envelope function, etc. (Hendriyana et al., 2018).

In summary, detection algorithms seek to determine the predicted value (PV) of the $\mathrm{CF}$ at some point and compare it with the current value (CV) measured in the $\mathrm{CF}$ at the same point. The $\mathrm{CV} / \mathrm{PV}$ relation allows identifying possible events if that exceeds a predefined threshold (THR) (Allen, 1982). Withers et al. (1998) reviewed some of the detection algorithms and found that none of them are optimal for all seismic event scenarios.

In this work, we use the pioneering and well-known $S T A / L T A$ algorithm (Allen, 1978), (Allen, 1982) and (Baer and Kradolfer, 1987). In this methodology, CV and PV values are calculated using the absolute amplitude average in time windows called shorttime average (STA) and long-term average (LTA). The STA's resulting value is sensitive to abrupt changes in the amplitude, while the LTA's give an approximate value of the 


\section{INTRODUCTION}

A

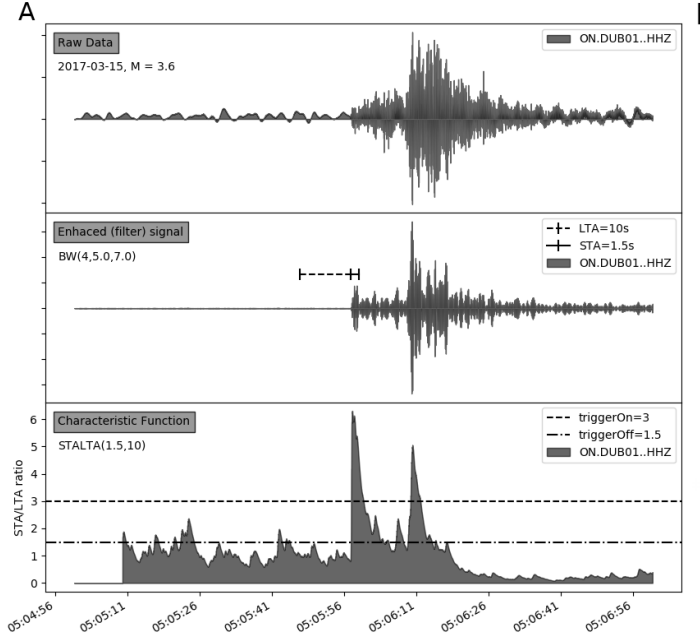

B

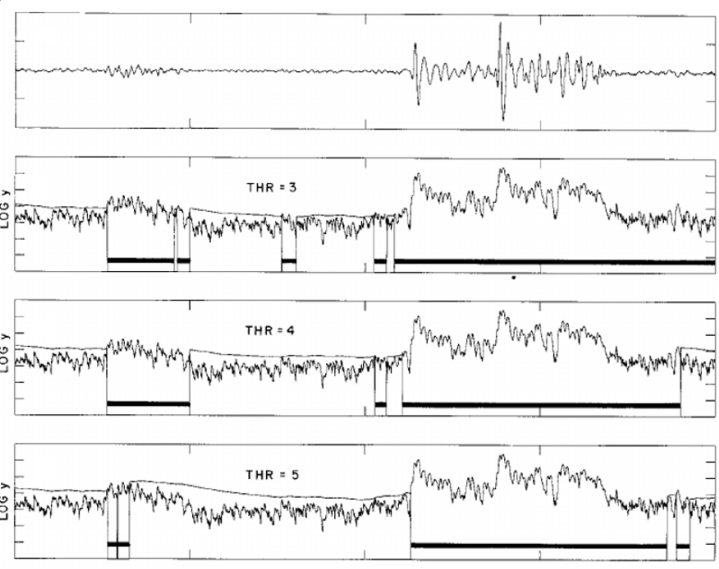

Figure 1.1: STA/LTA detector - A: Filtering and STA/LTA characteristic function from an event with magnitude 3.6 registered in DUB01 on 2017-03-15. B: Characteristic function and detection range using different thresholds, figure taken from Allen (1982)

amplitude of the seismic noise. The relation between STA and LTA values is used as $\mathrm{CV} / \mathrm{PV}$; when this relation exceeds a configured threshold (THR), the algorithm sends a new detection. The STA and LTA values are normally calculated using the CF, although they can also be calculated directly on the signal.

In Figure 1.1.A, we show an example of CF calculation; first, in the upper panel, we produce an enhanced signal applying a Butterworth BW(4,5.0,7.0) (four poles, five and seven $\mathrm{Hz}$ as low and high corner frequencies) to an event registered on DUB01 station. In the middle panel, we show LTA and STA windows, these go through all data, but we show the position where the point between windows matches with de $\mathrm{P}$ arrival. At this position relation between $\mathrm{CV}$ and $\mathrm{PV}$ take the maximum value. In the bottom panel, $\mathrm{CF}$ overpass the detection threshold called triggerOn for both $\mathrm{P}$ and $\mathrm{S}$ arrivals. We must configure two thresholds; triggerOn to send detections when CF overpasses it and triggerOff to activate the detection system when de CF takes values lower than it.

In Figure 1.1-B (taken from Allen (1982)), in the upper panel, the author presented a window that includes two events. In the three bottom panels, he showed detector performance using different triggerOn values. First, using triggerOn or "THR=3", both events were detected but also two false picks were produced. Using "THR=4" still generating one false pick. Finally using "THR $=5$ " only real detections were sent. 
The above shows the importance of a suitable configuration for detector parameters.

\section{$1.2 \quad$ Picker}

The picker must be executed as a step after detecting the seismic event and have to determine the onset times with significant accuracy. Therefore, the difference between the detection and picking is the accuracy when identifying the onset time. Many authors have proposed algorithms to use as an automatic picker; the Akaike information criteria or AIC method (MAEDA, 1985), the Autoregressive-Akaike information criteria (ARAIC) method (Sleeman and van Eck, 1999) and (Leonard, 2000), Wavelet Transform (CWT) (Zhang et al., 2003), and advanced techniques like artificial neural networks (Gentili and Michelini, 2006), fuzzy logic theory (Chu and Mendel, 1994) and higherorder statistics (Saragiotis et al., 1999).

In this work, we used an automatic P-phase picker based on AIC developed by Akaike (1998). The AIC method uses the assumption that a time series with an established duration can be divided into two sub-windows with pseudo-stationary time series (MAEDA, 1985) and (Sleeman and van Eck, 1999). The last means that both time series have some invariant statistical properties. Using the previous principle, a window with a seismic signal has different values for statistical properties than a window that includes only noise. The algorithm divides data using a rinning point that separates data into two windows. The algorithm analyzes the entire time series calculating the variance of both windows by modifying the middle point, which goes through all data. The time corresponding to the onset time matches with the point that best separates the window that includes only noise from the window containing the seismic signal; this corresponds to the lower AIC value.

MAEDA (1985) calculated the CF resulting from AIC directly from the signal without using an adjustment with the autoregressive method. The author proposed the following:

$$
A I C(k)=k \cdot \log (\operatorname{var}(x[1, k]))+(N-k-1) \cdot \log (\operatorname{var}(x[k+1, N]))
$$

Where the AIC is calculated for a seismogram $x$, of length $N$ and $k$ a point that take values through all $N$ samples. 


\section{INTRODUCTION}

The global minimum of the $\mathrm{CF}$ calculated by AIC will be found when the $\mathrm{k}$ point agrees with the onset time. It is essential to select fraction of the seismogram, including $\mathrm{P}, \mathrm{S}$ or both wave arrivals. The AIC algorithm does not guarantee that global minimum indicates onset when working with a low signal-to-noise ratio (SNR) (Zhang et al., 2003).
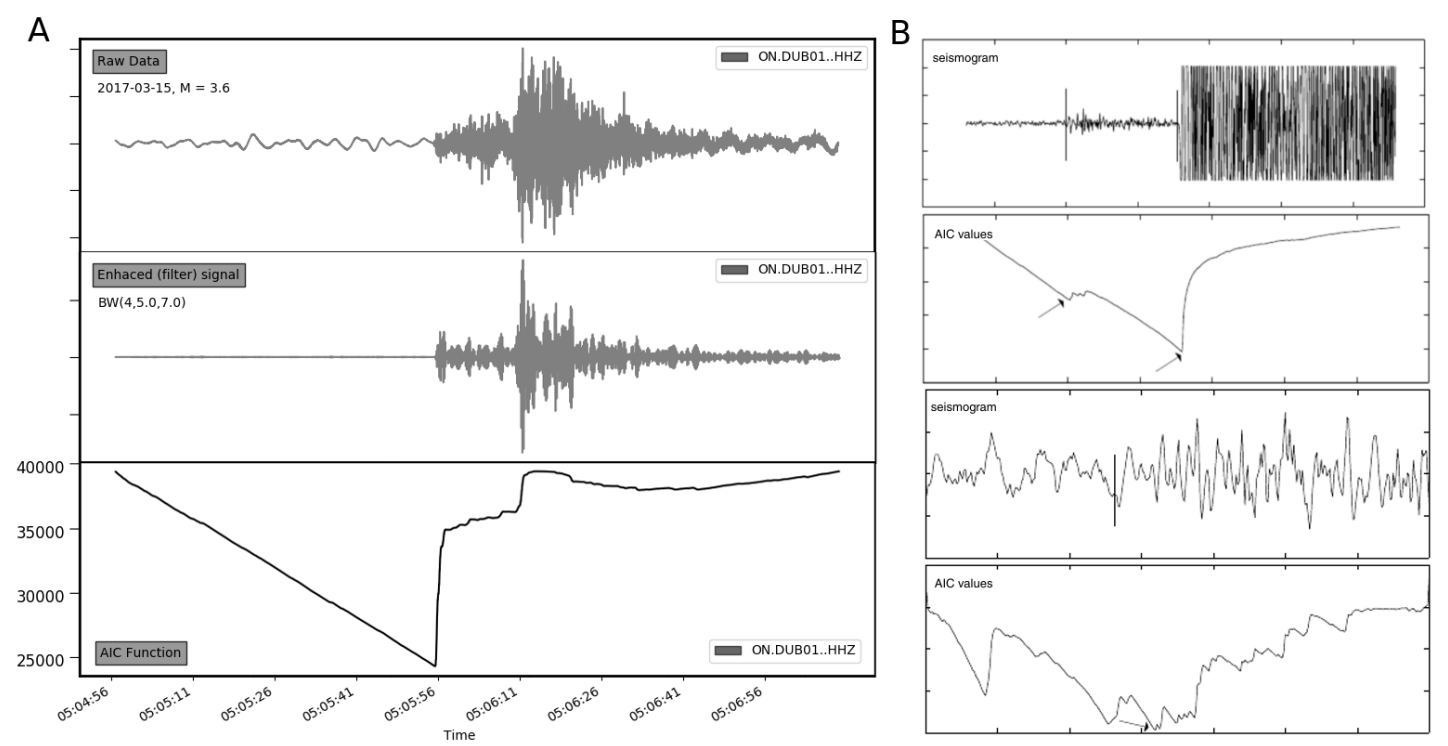

Figure 1.2: AIC picker - A: Filtering and AIC characteristic function from event registered with DUB01 station. B: Signal and AIC characteristic function for two events window and only noise window, figures taken from Zhang et al. (2003)

In Figure 1.2 A, we present a signal that includes an event registered in DUB01 station, the enhanced signal after filtering with a Butherwoth BW(4,5.0,7.0), and the $\mathrm{CF}$ after applying AIC. In the lower panel, the AIC function shows the global minimum that matches the $\mathrm{P}$ arrival and local minimum with $\mathrm{S}$ arrival. In Figure $1.2 \mathrm{~B}$, we present two examples extracted from Zhang et al. (2003), first a window with two earthquakes and the associated AIC function where the global minimum was sent, and the other arrival was lost. Second a window with inadequate filtering processes that hides the event, the AIC algorithm sends a false pick that agrees with the minimum. Both examples show the importance of choosing the correct filter and window to run the AIC function. 


\subsection{Parameter optimization:}

The detection and picker algorithms are commonly applied to pre-filtered signals usually using band-pass filters; this filter is applied to reduce seismic noise and increase the signal-to-noise ratio (SNR) of the seismic events. The filtering process is equivalent to the methodologies for producing characteristic functions (Withers et al., 1998). In this study we use Butterworth (BW) filters.

Results from $S T A / L T A$ detection algorithm are sensitive to parameters such as the thresholds and the $S T A / L T A$ windows lengths. Likewise, the AIC picker algorithm is mainly sensitive to the selection of the time window.

Therefore, a rigorous search for the appropriate set of parameters for the filtering, detection, and picker processes is recommended.

On the other hand, to evaluate automatic picks, we compared them against manual picks. If there is a comparable, we evaluate the automatic pick as real and, if not, this would be a false pick. This method is presented in detail in the paper.

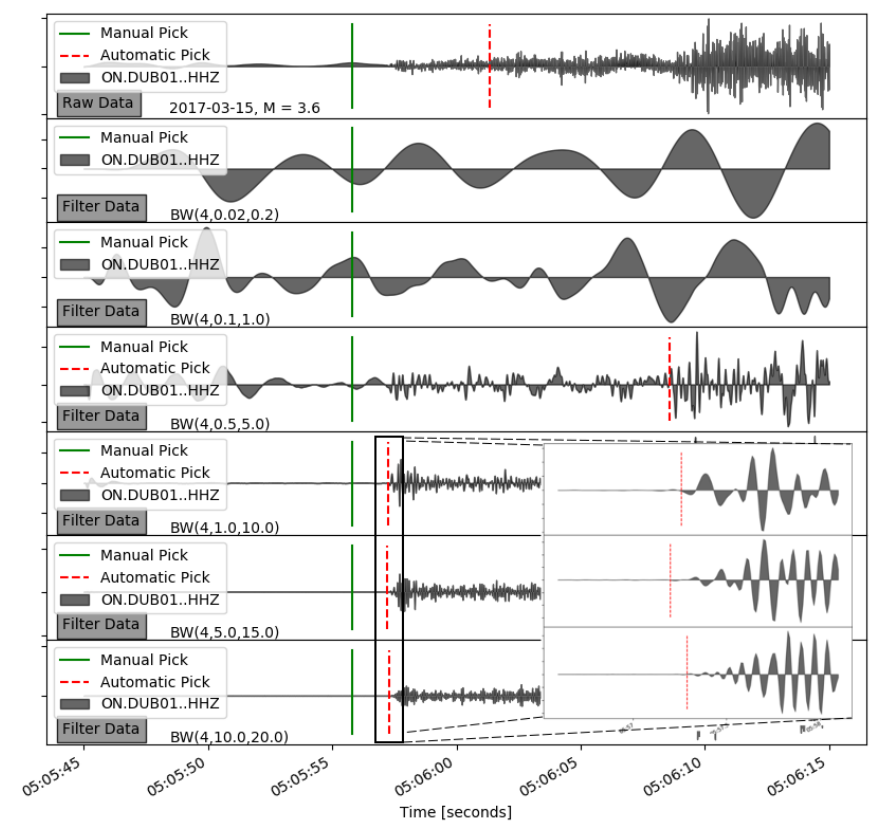

Figure 1.3: Optimization example - Processing the same event signal with seven different parameter sets. Changes in Butterworth filter are shown.

In Figure 1.3 we present seven results after running the picker algorithm using different BW filter configurations using data from a real event with magnitude 3.6 


\section{INTRODUCTION}

recorded by DUB01 station on 2017-03-15. For raw data, the automatic pick has a shift to the real phase arrival; using low-frequency filters $\mathrm{BW}(4,0.02,0.2)$ and $\mathrm{BW}(4,0.1,1.0)$, the automatic processing system failed to find the arrival. With medium-frequency filter BW(4,0.5,5), a false $\mathrm{P}$ phase was marked on the $\mathrm{S}$ phase arrival. Finally, with all high-frequency filters, the system selected the arrival close to the real onset time. In the zoom window, we present differences between automatic picks produced with high-frequency filters. The above shows us that we can divide the optimization process into two steps; first, to find arrivals close to the real onset time and second to improve the quality of these arrivals getting as close as possible to the real onset time.

The parameter optimization seeks to optimize the results for as many events as possible by increasing the number of real automatic picks. There is no widely used methodology for optimizing these parameters; therefore, some seismology centers have chosen to perform this optimization manually. Unfortunately, manual optimization can be challenging due to factors such as an increased number of false picks, complexity in finding suitable parameters for a large sample of events with different characteristics, and the requirement to adjust parameters by station or group of stations. Therefore, seismology centers usually use automatic processing systems without previously applying a parameter optimization process.

Some authors have proposed optimization methods e.g., Olivieri et al. (2007), Vassallo et al. (2012). Others provide lists with parameters already adjusted Pechmann (2006). We use a similar approach used by Vassallo et al. (2012) on this work.

The parameter space is explored using grid search (Bergstra et al., 2011b), or Bayesian (Wu et al., 2019) as optimization methodologies, seeking the best set of parameters. An iterative process is done by running the detector and picker algorithms changing the parameters; this method seeks to maximize an objective function that evaluates the results comparing automatic picks with the manual picks previously selected.

\subsection{Objective function}

The objective function is essential for optimization. Vassallo et al. (2012) proposed a function that depends on the ratio of the time difference between automatic and manual picks versus the manual pick's uncertainty. In this work, we use Recall and the 
F1-score accuracy-test (Sokolova and Lapalme, 2009) as objective functions. F1-score is calculated from the test's precision and recall.

$$
F 1=2 *\left(\frac{\text { precision } * \text { recall }}{\text { precision }+ \text { recall }}\right)
$$

Where precision is the portion of real picks identified among all identified picks, including false ones, Recall is the portion of real picks identified among all real picks, including those that our model does not identify. A precision of 0.7 means that $70 \%$ of all automatic picks are real (30\% are false picks). A recall of 0.8 means that $80 \%$ of the all real picks were found ( $20 \%$ of real picks were missed).

To calculate these metrics, we use:

$$
\begin{gathered}
\text { Precision }=\frac{T P}{T P+F P} \\
\text { Recall }=\frac{T P}{T P+F N}
\end{gathered}
$$

Where TP are the true positives or real picks identified, FP are the false positives or false picks, and FN are the false negatives or real unidentified picks. See Figure 1.4

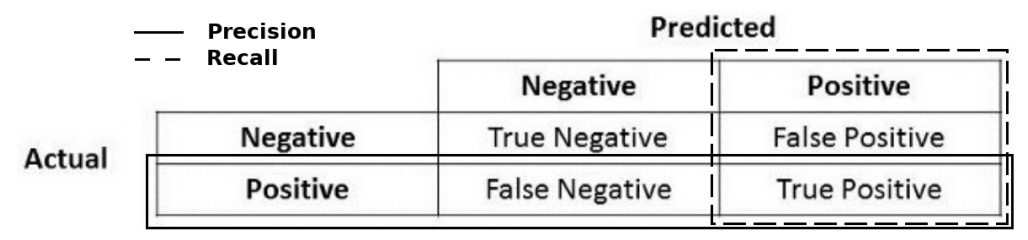

Figure 1.4: Precision and Recall - Apply to our study, actual are the manual picks and predicted the automatic picks.

The maximum value of the F1-score is one and means the best possible result. It is essential to remark that increasing precision often reduces recall and vice versa. The above is applied to this work by the fact that increasing real picks (true positives) will increase false picks (false positives). Therefore, the methodology must find an optimum trade-off between real and false picks.

\subsection{Grid Search}

The most common strategy for doing a global search in parameter space is grid-search (Bergstra et al. 2011b). In this methodology, ranges values for each parameter are selected, and all combinations between them are tested (Figure 1.5). The number of tests 


\section{INTRODUCTION}

increases exponentially with the number of parameters; therefore, grid search is inefficient for a large number of parameters being optimized (Wu et al., 2019). Some authors have proposed improved Grid-search methodologies such as doing a first exploration with large steps looking for ranges with good results, then followed by a more detailed search over these areas (Wang, 2014), or using bilinear Grid search method (Hsu et al. 2003). In this study, we implemented the standard Grid search where all combinations of the first parameter are tested and for each value, all combinations for the second parameter are tested, and so on. We use fixed steps when running Grid-search.

Grid search methodology has a high learning accuracy (Taijia Xiao et al., 2014) and allows the easy understanding of parameter optimization due to its conceptual simplicity. Grid search is easy to implement with trivial parallelization because the optimization process is done for each parameter independently, therefore, we decided to parallelized it. Finally, grid search allows us to compare performance and results versus other methodologies such as Bayesian optimization (Betrò, 1992).
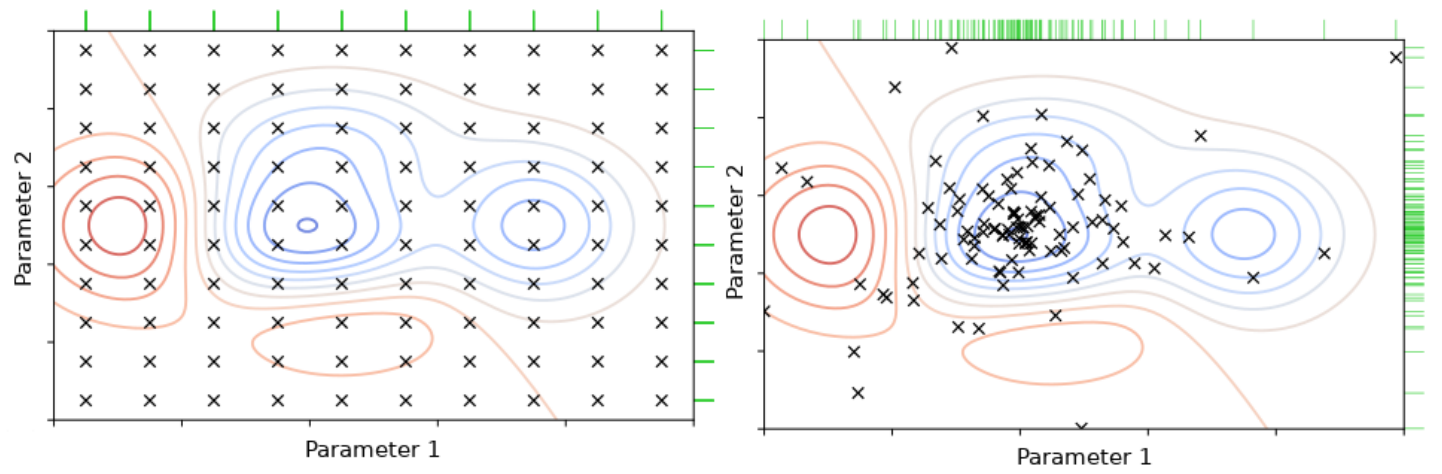

Figure 1.5: Grid-search and Bayesian - Two dimensional space exploration with Grid-search to the left and Bayesian to the right.

\subsection{Bayesian}

Bayesian optimization, also known as Sequential Model-based Global Optimization (Mockus et al., 2014), is a statistical technique for finding the extrema of a function with an unknown form (black box) called objective function. This method is very useful for function expensive to evaluate.

Contrary to techniques such as the grid-search or random-search, it produced a assumption of the objective function and uses previous function evaluations as evidence 
to efficiently choose the next evaluation point (Snoek et al., 2012). Using this strategy going through all parameter space is not necessary (Figure 1.5), and a lower number of function evaluations are sufficient. This methodology uses previous function evaluations to build a surrogate function, which approximates the probability distribution of the objective function (PDF). The drawback of this strategy is the bias between the PDF and the final result, this difference increase when the evidences are insufficient.

There are several strategies to build the surrogate function but it is common to use a Gaussian Process (GP) (Bergstra et al., 2011a).

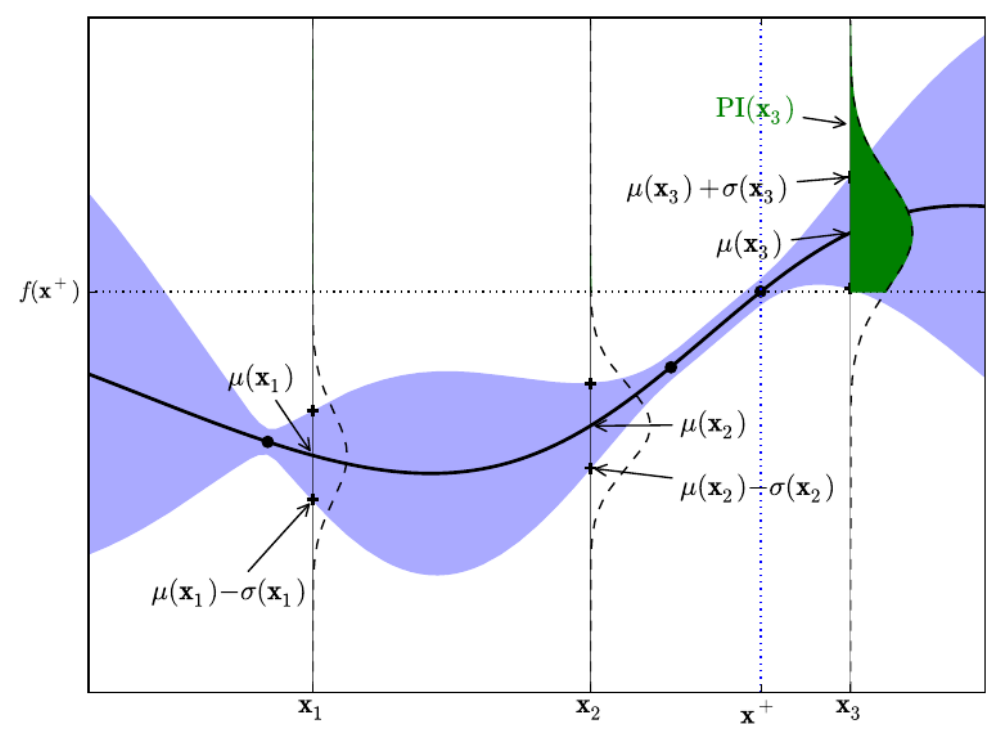

Figure 1.6: 1D Gaussian process - Solid line is the surrogate mean prediction using GP given three function evaluations plotted with black dots. The purple shaded area exhibit the variance at each point. Gaussians constructed with mean and standard deviation at $x_{1}$, $x_{2}$ and $x_{3}$ represent predictions at these points. Objective function at $X^{+}$is the maximum observation and green shaded area show the probable improvement. Figure taken from Brochu et al. (2010).

GP could be compared with a function but instead of result in a scalar $f(x)$ for any $x$, it produces the mean and variance of a normal distribution over possible values of function $f$ evaluated at $x$ (Brochu et al., 2010). See Figure 1.6.

Figure 1.6 shows an example of one iteration following the Bayesian optimization process. At each iteration, evidences and variances (calculated with GP) are essential to build the probability of improvement PI presented with the green area. Points with 


\section{INTRODUCTION}

surrogate function values higher than $f\left(x^{+}\right)$have a probability of improvement such as $x_{3}$.

After defining how Bayesian optimization outlines the objective function using observations and GP processes, it is essential to explain how this methodology chooses the optimum value to execute the following observation. A new function called "acquisition function" is built using the mean and variance from the surrogate function. Maximum values of the acquisition function are promising points to find the extrema of the objective function. The result of testing at the suggested point is used to update the surrogate function.

The exploration-exploitation trade-off is the key to a good acquisition process. Exploration is centered in points where the variance of surrogate function is large, meanwhile exploitation in points where the mean of surrogate function is large. Several methods to build the acquisition function try to balance both exploring and exploiting, it is common to use Expected Improvement (EI) or Probability of Improvement (PI) (Jones, 2001). Figure 1.6 show a typical PI implementation.

Figure 1.7 shows the Bayesian optimization iterative process using GP. In the top panel, two observations and variances are used to build the first surrogate function as an approximation of the PDF, the acquisition function is build using PI and is maximized to find the point for new observation. In the middle panel the new observation was added as evidence and acquisition function is updated to find new maxima and therefore a new observation point. The bottom panel shows the final iteration where, using all evidence, the PDF maximum is found. 

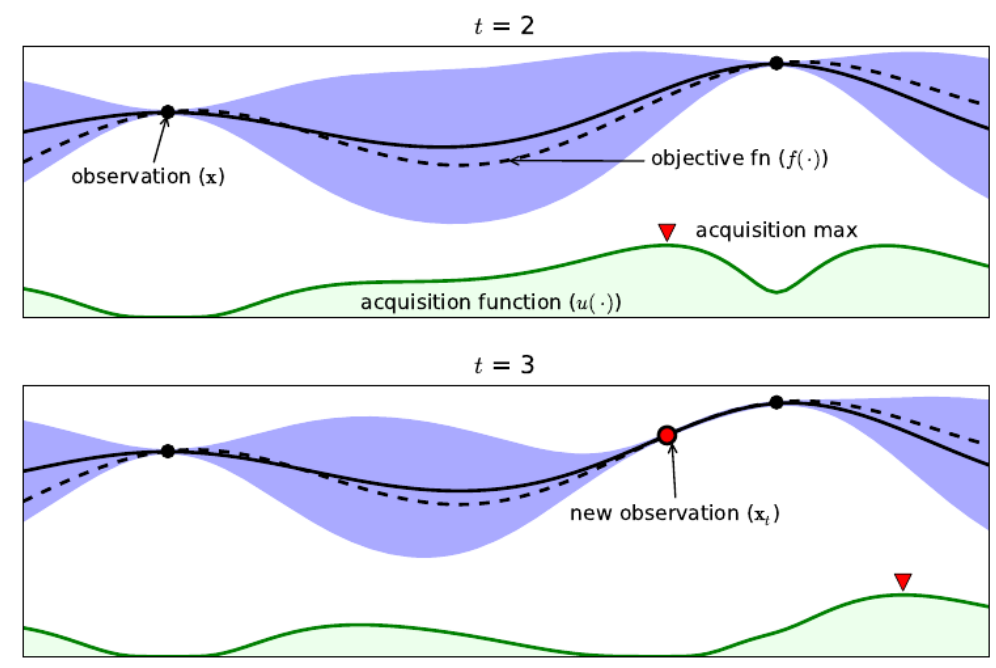

$t=4$

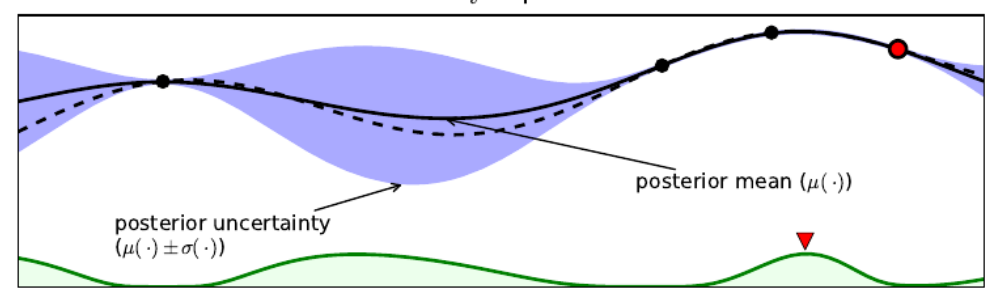

Figure 1.7: 1D Gaussian process iteration - Dashed and solid lines are the objective function and GP approximation respectively, Purple shaded zones show the variance from GP approximation, green shaded zones the acquisition function and red triangle the maximum of acquisition function. Figure taken from Brochu et al. (2010). 


\section{Paper}

In this section, the paper submitted to the seismology journal "Bulletin of the Seismological Society of America" is attached. The article was submitted on April 16, 2021. The paper is currently under revisions by the editor and reviewers.

The paper, in section one, briefly introduces some of the principal concepts for detector, picker, global optimization, objective function, grid search, and Bayesian presented in section 1 of this document. Next, we present maps and plots to show stations and events extracted from RSNC and RSBR networks.

In section two, all methods used are presented, including the code picktune which was the principal utility used during this study. A schema of the workflow proposed to do the parameter optimization for detector and picker parameters is presented in the methodology section. Pick evaluation method based on comparing manual and automatic picks using step functions is presented next to some typical examples found during this study.

Section three includes results after using grid search and Bayesian optimization. This section presents results for the DIAM station as an example of results obtained for all stations optimized. Plots including the total number of automatic picks for both networks after and before optimization are included.

Section four presents the discussion of results for all stations and both grid-search and Bayesian optimization, including the comparison between them. Maps and plots with automatic locations calculated using automatic picks after optimization are presented.

Finally, the main conclusions are presented. 


\title{
Parameter optimization of automatic phase detection and picking algorithms - Application in São Paulo University Seismological Center and Colombian National Seismic Network.
}

\author{
Camilo Muñoz Lopez ${ }^{\mathrm{a}}$, Daniel Siervo ${ }^{\mathrm{b}}$, Marcelo Assumpção ${ }^{\mathrm{a}}$, Viviana Dionicio ${ }^{\mathrm{b}}$ \\ ${ }^{a}$ Institute of Astronomy, Geophysics and atmospheric Sciences, University of Sao Paulo \\ ${ }^{b}$ Seismological Network - Servicio Geológico Colombiano
}

\begin{abstract}
Two methodologies, Grid-search and Bayesian optimization, were used to optimize the automatic detection and phase picking parameters in SeisComP. These methodologies were tested using a set of stations selected from two seismological networks the Brazilian Seismic Network (RSBR) and Colombian National Seismological Network (RSNC).

After comparing manual and the initial automatic locations, we found numerous missing events and others with low-quality locations in automatic databases. We selected 508 manual events from 2017/01/01 to 2020/07/31 in Brazil, and 532 manual events from 2019/02/01 to 2019/02/15 near to Bucaramanga Nest in Colombia, as training data sets for the optimization process.

A code was implemented to use an iterative grid-search to optimize the picking parameters. In addition, the Optuna Python package was used to implement the Bayesian optimization. Selected events were used as a training set, and an iterative process according to the Bayesian method was used. The results of both methodologies were compared.

Both methodologies showed excellent results. Grid-search allowed us to perform a complete analysis of the results examining the entire space of parameters. However, Grid-search lose efficiency while increasing the number of parameters being optimized. On the other hand, the Bayesian algorithm is computationally more efficient by not exploring the entire parameter space.

After the optimization process, automatic picks increases by $78 \%$ and $56 \%$ for RSBR and RSNC, respectively. The number of locations calculated using new automatic picks doubled the automatic locations determined by the systems before the optimization process for both networks.

Seismological centers could implement methodologies such as Grid-search or Bayesian algorithm to improve their automatic processing systems. Besides, the standardization of these methodologies would help to make easier their implementation.
\end{abstract}

Keywords: Automatic processing system, Detection, Picking, Optimization, Grid-search and Bayesian.

\section{Introduction}

The increasing amount of seismic data recorded and stored by seismological networks in Latin America makes manual processing difficult. Manual picking of seismic phases has been the methodology most used in seismological centers, but it is a time-consuming process and includes the error associated with the person's expertise in charge (Leonard (2000)). A prompt and good quality response is a priority for most seismological networks. Accurate and efficient analysis of seismic data requires the implementation of automatic processing algorithms. Therefore, the reliability 
and quality of the results produced automatically have become a critical requirement for the seismological network's.

The arrival picking of the seismic phases is the first step of automatic processing. The most accepted methodologies use variations in amplitude or frequency between the seismic noise and the earthquake signal (Withers et al. (1998)) to automatically identify the seismic phases. In this article, we use the approach used by Allen (1982), separating the arrival picking into two sub-processes, the detection to find the seismic phases and the picker to estimate the onset time.

\section{Detector}

Detection seeks to identify arrivals of seismic phases without the purpose of accurately determining their onset times. Several algorithms have been proposed for the automatic detection of events Allen (1978) Baer and Kradolfer (1987). Most authors calculate a characteristic function $(C F)$ which is a substitute function calculated by applying to the original time series some operation to highlight the onset of $\mathrm{P}$ or $\mathrm{S}$ waves. The $C F$ can be calculated using filtering, absolute amplitude, power, polarization indicator, envelope function, etc. (Hendriyana et al. (2018)).

In summary, detection algorithms seek to determine the expected CF's value at some point (PV) and compare it with the current value measured in the CF (CV). The CV/PV relation allows identifying possible events if that exceeds a predefined threshold (THR) Allen (1982). Withers et al. (1998) reviewed some of the detection algorithms and found that none of them are optimal for all seismic event scenarios. All methodologies to build a CF have some weaknesses. For example, using $\mathrm{CV} / \mathrm{PV}$ needs a variation in amplitude and ignore changes in frequency. Therefore, we can miss detections for small events or data series with high noise using this methodology.

In this work, we use the pioneering and well-known $S T A / L T A$ algorithm Allen (1978), Allen (1982) and Baer and Kradolfer (1987). In this methodology, CV and PV values are calculated using the absolute amplitude average in time windows called short-time average (STA) and longterm average (LTA). The STA's resulting value is sensitive to abrupt changes in the amplitude, while the LTA's give an approximate value of the amplitude of the seismic noise. The relation between STA and LTA values is used as CV/PV; when this relation exceeds a configured threshold (trigOn), the algorithm sends a new detection. The STA and LTA values are normally calculated using the $\mathrm{CF}$, although they can also be calculated directly on the time series.

\section{Picker}

The picker must be executed as a step after detecting the seismic event and have to determine the onset times with significant accuracy. Therefore, the difference between the detection and picking is the accuracy when identifying the onset time. Many authors have proposed algorithms to use as an automatic picker; the Akaike information criteria or AIC method (MAEDA (1985)), the Autoregressive-Akaike information criteria (AR-AIC) method (Sleeman and van Eck (1999) and Leonard (2000)), Wavelet Transform (CWT) (Zhang et al. (2003)), and advanced techniques like artificial neural networks (Gentili and Michelini (2006)), fuzzy logic theory (Chu and Mendel (1994)) and higher-order statistics (Saragiotis et al. (1999)).

In this work, we used an automatic P-phase picker based on AIC developed in 1971 (Akaike (1998)). This criterion seeks the best fit for the observed data through an autoregressive (AR) statistical method. AR-AIC method works with the assumption that a time series with an established duration can be divided into two sub-windows with pseudo-stationary time series (MAEDA (1985) and Sleeman and van Eck (1999)). The last means that both time series have some invariant statistical properties. Using the previous principle, a window with a seismic signal has different values for statistical properties than a window that includes only noise. The algorithm analyzes the entire time series by modifying the point that separates the two sub-windows. The time corresponding to the onset time is the point that best separates the window that includes 
only noise from the window that contains the seismic signal; this point corresponds to the lower AIC value.

MAEDA (1985) calculated the CF resulting from AIC directly from the signal without using an adjustment with the autoregressive method. Maeda proposed the following:

$$
A I C(k)=k \cdot \log (\operatorname{var}(x[1, k]))+(N-k-1) \cdot \log (\operatorname{var}(x[k+1, N]))
$$

Where the AIC is calculated for a seismogram $x$, of length $N$ and $k$ a point that take values through all $N$ samples.

The global minimum of the CF calculated by AIC will be found when the k point agrees with the onset time. It is essential to select only the fraction of the seismogram, including $\mathrm{P}$ or $\mathrm{S}$ wave arrival. The AIC algorithm does not guarantee that global minimum indicates onset when working with a low signal-to-noise ratio (SNR) (Zhang et al. (2003)); therefore, it is essential to choose an appropriate time window.

\section{Global optimization:}

The detection and picker algorithms are commonly applied to pre-filtered signals; this filter is applied to reduce seismic noise and increase the signal from seismic events or, in other words, to improve the SNR value. The filtering process is equivalent to the methodologies for producing characteristic functions (Withers et al. (1998)). In this study we use Butterworth (BW) filters.

Results from $S T A / L T A$ detection algorithm are sensitive to parameters such as the thresholds and the $S T A / L T A$ windows lengths. Likewise, the AIC picker algorithm is mainly sensitive to the selection of the time window.

Therefore, a rigorous search for the appropriate set of parameters for the filtering, detection, and picker processes is recommended. This operation seeks to optimize the results for as many events as possible by increasing the number of automatic picks. There is no widely used methodology for optimizing these parameters; therefore, some seismology centers have chosen to perform this optimization manually. Unfortunately, manual optimization can be challenging due to factors such as an increased number of false picks, complexity in finding suitable parameters for a large sample of events with different characteristics, and the requirement to adjust parameters by station or group of stations. Therefore, seismology centers usually use automatic processing systems without previously applying a parameter optimization process.

Some authors have proposed optimization methods (e.g., Olivieri et al. (2007), Vassallo et al. (2012)). Others provide lists with parameters already adjusted (Pechmann (2006)). We use a similar approach used by Vassallo et al. (2012) on this work.

The parameter space is explored using grid search Bergstra et al. (2011b), or Bayesian Wu et al. (2019) as optimization methodologies, seeking the best set of parameters. An iterative process is done by running the detector and picker algorithms changing the parameters; this method seeks to maximize an objective function that evaluates the results comparing automatic picks with the previously selected manual picks.

\section{Objective function:}

The objective function is essential for optimization. Vassallo et al. (2012) proposed a function that depends on the ratio of the time difference between automatic and manual picks versus the manual pick's uncertainty. In this work, we use Recall and the F1-score accuracy-test (Sokolova and Lapalme (2009)) as objective functions. F1-score is calculated from the test's precision and recall.

$$
F 1=2 *\left(\frac{\text { precision } * \text { recall }}{\text { precission }+ \text { recall }}\right)
$$


Where precision is the portion of real picks identified among all identified picks, including fake ones, the recall is the portion of real picks identified among all real picks, including those that our model does not identify. To calculate these parameters, we use:

$$
\begin{gathered}
\text { Precision }=\frac{T P}{T P+F P} \\
\text { Recall }=\frac{T P}{T P+F N}
\end{gathered}
$$

Where TP are the true positives or real picks identified, FP are the false positives or fake picks, and $\mathrm{FN}$ are the false negatives or real unidentified picks.

The maximum value of the F1-score is one and is the best possible result. It is essential to remark that increasing precision often reduces recall and vice versa. The above is applied to this work by the fact that increasing real picks (true positives) will increase false picks (false positives). Therefore, the methodology must find an optimum trade-off between real and false picks.

\section{Grid Search:}

The most common strategy for doing a global search in parameter space is grid-search (Bergstra et al. (2011b)). In this methodology, ranges values for each parameter are selected, and all combinations between them are tested. The number of tests increases exponentially with the number of parameters; therefore, grid search is inefficient for a large number of parameters being optimized (Wu et al. (2019)).

However, grid search has a high learning accuracy (Taijia Xiao et al. (2014)) and allows an easy understanding of the global optimization results due to its conceptual simplicity. Furthermore, this method is easy to implement with trivial parallelization because the optimization process is done for each parameter independently. Finally, grid search allows us to compare performance and results versus other methodologies such as Bayesian optimization (Betrò (1992)).

\section{Bayesian:}

Bayesian optimization, also known as Sequential Model-based Global Optimization Mockus et al. (2014), is a statistical technique for finding the extrema of a function with an unknown form (black box). In this study, we want to find the maximum of the objective function. This method is handy for functions expensive to evaluate.

Contrary to techniques such as the grid-search or random-search, it calculates a prior model of the objective function, and using function evaluations as evidence, efficiently chooses the next evaluation point Snoek et al. (2012). Using this strategy, going through all parameter space is unnecessary; therefore, this requires just a few function evaluations.

This methodology uses previous function evaluations to build a surrogate function, which approximates the objective function's probability distribution. There are several strategies to build the surrogate function, but it is common to use a Gaussian Process (GP) Bergstra et al. (2011a).

It is essential to explain how this methodology chooses the optimum value to execute the next observation. The surrogate function is analyzed with the acquisition function; from this analysis, promising points to find the extrema are obtained. The result of evaluating the objective function at the suggested point is used to update the surrogate function. It is common to use Expected Improvement (EI) or Probability of Improvement (PI) to build the acquisition function Jones (2001). 


\section{Methodology}

In this work, two data sets were used, the first one with earthquakes located in Brazil and the second one with earthquakes belonging to a much smaller and very active area known as the Bucaramanga Nest in Colombia (Prieto et al. (2012)). For Brazil, the southern region was selected for having the highest number of stations and earthquakes. All events manually located by the Sao Paulo university seismological center (IAG-USP) from January 2017 to June 2020 were selected (figure 1). In this period, 508 events were selected recorded in 52 stations.

Eight broad band stations closest to the Bucaramanga nest (Prieto et al. (2012)) were selected from seismological network in Colombia (Colombiano (1993)). 532 well-recorded earthquakes and located manually by the RSNC from February 1 to 15, 2019, were used.

Data from Colombia allow us to evaluate our methodology's performance for a local network installed in a small area with high seismic activity (Zarifi and Havskov (2003)), while those from Brazil represent the performance in regional networks with reduced seismicity (Bianchi et al. (2018)).
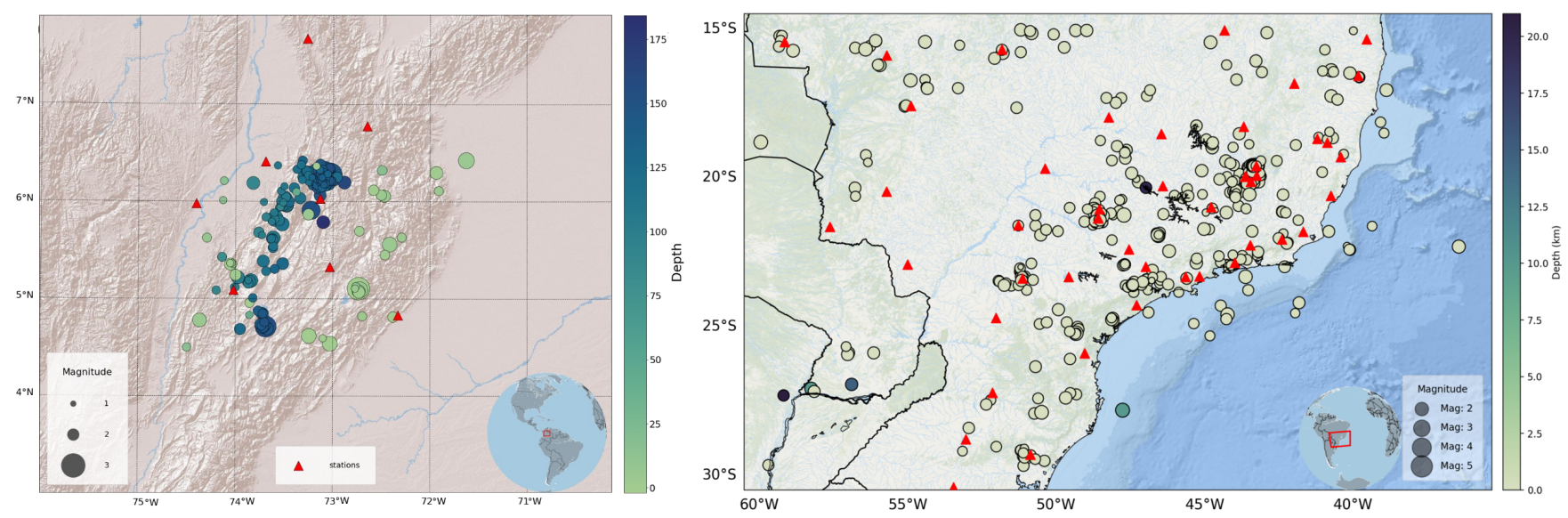

Figure 1: Maps with both Brazil and Colombia interest areas showing earthquakes and stations selected to use in the optimization process. Circles are epicenters from the manually revised catalogs.
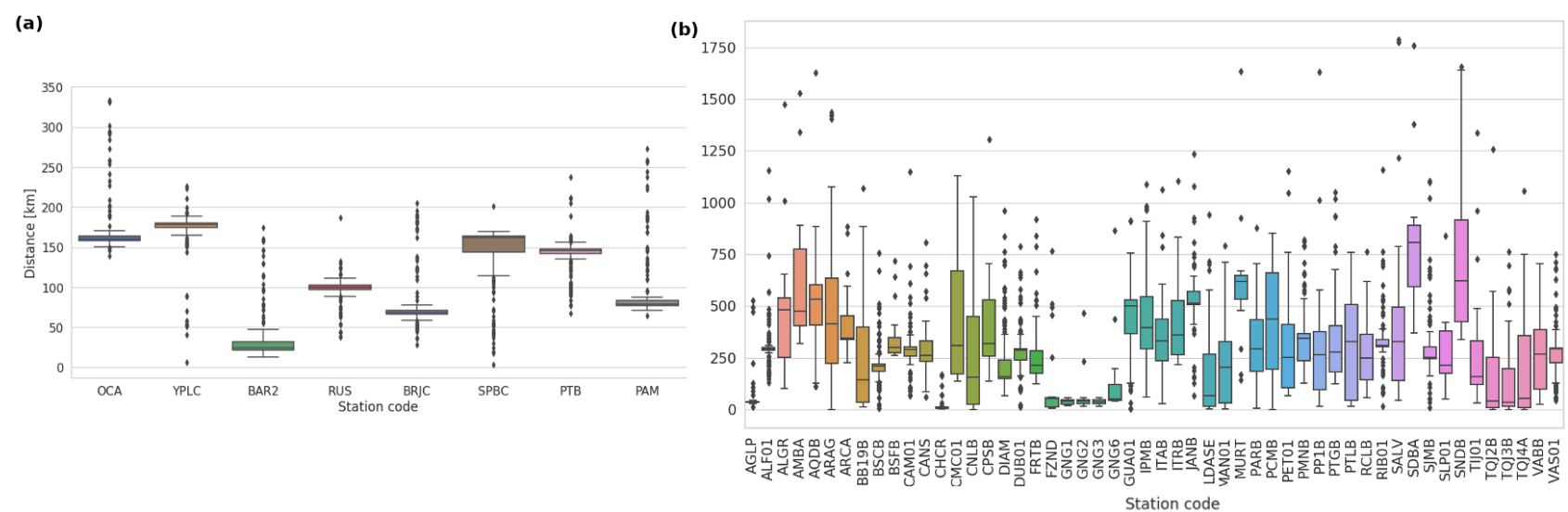

Figure 2: Hypocentral distances distribution for each station. (a) Colombia (b): Brazil

Figure 2 shows hypocentral distances distribution for each station for both Colombia and Brazil. For Colombia, the mean hypocentral distance close to $100 \mathrm{~km}$ presents a local network 
with concentrated seismicity. Stations with the highest and lowest hypocentral distances are OCA up to $330 \mathrm{~km}$ and BAR2 with $3 \mathrm{~km}$, respectively. Events selected for Colombia have a local magnitude (ML) range from 1.2 to 3.0. On the other hand, events selected in Brazil show a different hypocentral distance distribution with typical values from 0 to $1000 \mathrm{Km}$. Local magnitude values for selected events in Brazil go from 1.0 to 4.0.

A code called picktune that optimizes the automatic processing system was developed (Figure 3). Two methodologies were implemented in picktune; the first one using Grid-search and recall as the score and the second one Bayesian and F1 score. For RSBR both methodologies were applied using the same training data and results were compared. For RSNC, we decided only to use Bayesian optimization because of the low computing time required and good performance in the Brazilian case.

The procedure performed by picktune can be divided into five main steps:

Preparation of training data: Events were selected, including their manual picks; these data were used as the training data set. Bindings (configuration files for each station) were created, and 10 minutes waveforms around each manual pick from these events were extracted.

Initial evaluation: Performance of the automatic processing system was evaluated before the optimization; this was done by comparing manual versus automatic picks. This comparison allowed us to evaluate the current system's performance before optimization and will be the starting point to evaluate results to find improvements after the optimization process.

New trial parameters: A new set of parameters called a new "trial" is selected. The code picktune has implemented two methodologies to select this new trial; by exhaustive exploring with grid-search or using the more efficient Bayesian algorithm. Parameters from the detection and picking algorithms were included. For the detector, BW corner frequencies, STA and LTA windows lengths, and trigOn. For the picker, BW corner frequencies and the SNR. Picktune code allows adding new parameters involved in the detection and phase picking algorithms.

In this work, when grid-search is used, due to excessive increase in computing time not all parameter combinations were tested; the optimization was done in the sequence shown below; combinations for low an high BW detectors corner frequencies, combinations for STA/LTA windows lengths, values for trigOn, combinations for low an high BW picker corner frequencies and finally SNR values. Thus, in each stage the best parameter trial were selected and used for the next stage. Although this may be a weakness in the grid-search methodology for not testing all the combinations, it generated promising results at lower computation time.

Processing: Detector and picker algorithms are executed using the trial parameter previously selected by grid-search or Bayesian algorithms. Multiprocessing to run detector and picker algorithms for several waveforms at the same time was implemented.

Results evaluation: Resulting automatic picks were stored and compared with manual picks of the training set to calculate the objective value (recall or f1-score).

The iterative process that includes the selection of new trial parameters, processing, and result evaluation is what we call optimization. SeisComP (Weber et al. (2007)) was used as the principal software for the acquisition and data processing in this work. 


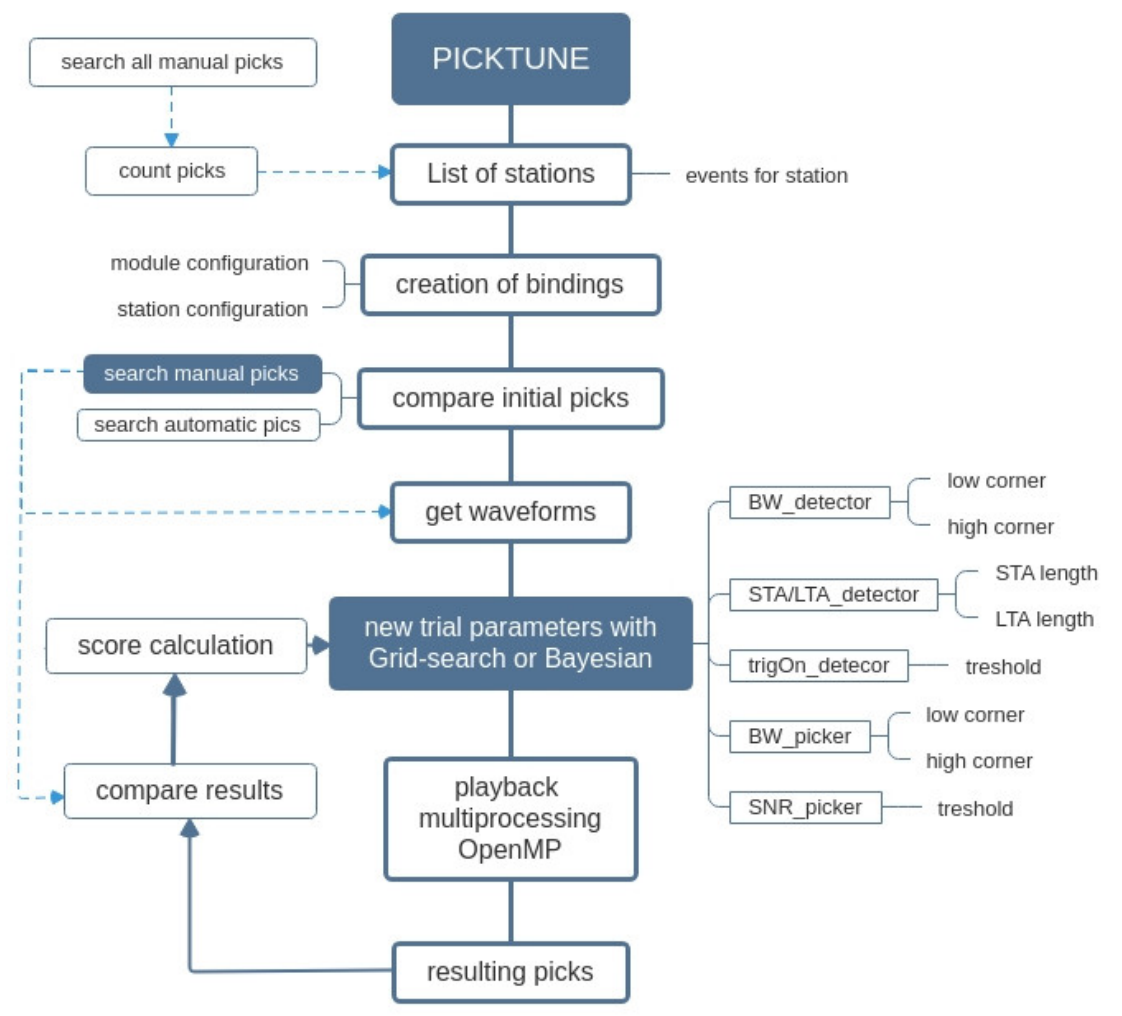

Figure 3: Diagram with the procedures performed by picktune code.

Picks obtained after each iteration must be evaluated; like other authors (Sleeman and van Eck (1999), Baer and Kradolfer (1987), Leonard (2000) and Chang et al. (2019)), we evaluated comparing difference between automatic and the manual picks onset-times. The whole optimization process is susceptible to this comparison process. The critical parameter to configure is the length of the associated window; if the difference between automatic and manual onset times is equal or less than this window, these picks are classified as "comparable". Figure 4-a shows three examples of automatic picks (red color) compared with manual picks (green color); analyzing the first example, we conclude that the association window must be wide enough to establish as "comparable" situations in which the difference between onset-times of the automatic and manual picks is a little higher than expected. The second case present the expected behavior where both manual and automatic picks are very close to each other; therefore, their association could be done with a small association window. In the third example, the manual pick has an error, in this situation, the association window must be large enough to find automatic picks close to the manual pick or close to the real onset time. Therefore, the identification window must be large enough to include automatic picks a bit far from manual picks, but it cannot be so large to include false picks. 

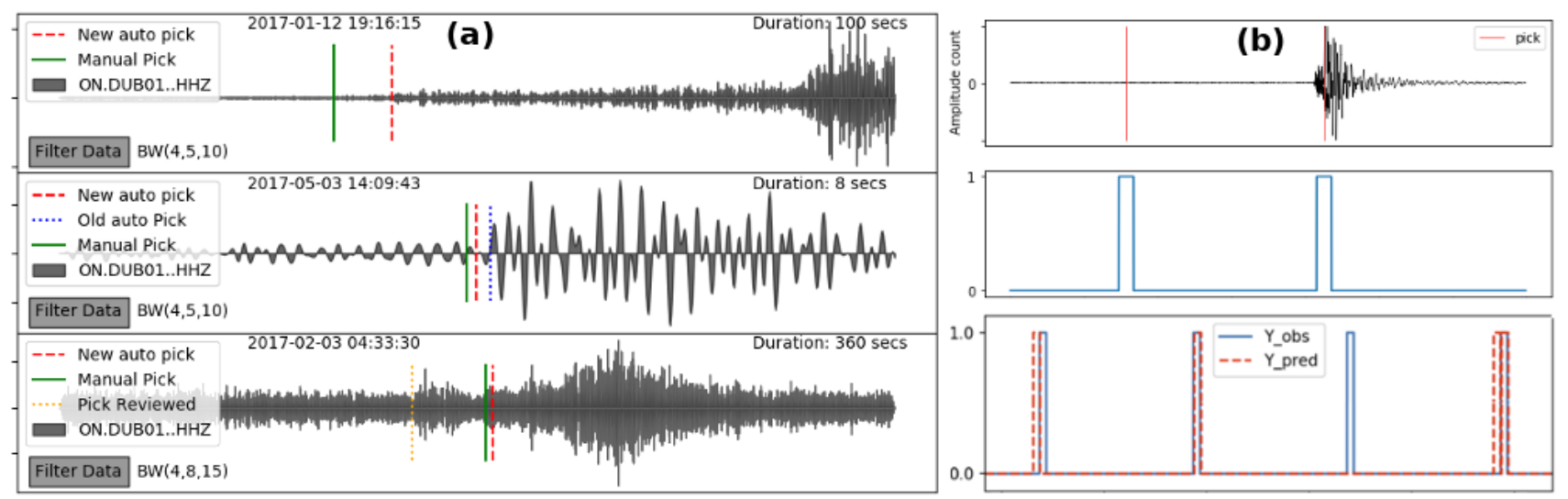

Figure 4: Pick comparison process. a: Examples with comparable manual and automatic picks for three different events. b: Step functions building. The figures show: at the top, a seismogram with two automatic picks, in the middle, the step function called "observed" for these picks, and in the bottom, the correlation between an observed and a predicted step functions.

Step functions were constructed, assigning one to all samples 0.5 seconds before and after each manual and automatic pick, and 0 outside this window. See figure 4-b. The observed function was build using automatic picks and predicted function with manual picks. These step functions were correlated to obtain true positive if steps from both functions match at least one point, false negatives for steps only in predicted function, false positives for steps only in observed function, and true negatives in places without any step. See blue and red step functions in figure 4-b.

The preceding was done to approximate pick optimization problem to a binary classification problem (Géron and Safari (2019)). A score was calculated for each trial parameter set and was finally used to choose the best trial.

Bayesian optimization was performed applying the Optuna python package (Akiba et al. (2019)) and using the Gaussian Process and the Expected Improvement as substitute and acquisition function. F1-score calculated from binary functions was used as the objective function. The F1-score obtained in each iteration and the corresponding trial parameter set were applied to the Bayesian optimization algorithm to suggest the new parameter set to evaluate. Two hundred iterations were used to optimize each station.

\section{Results}

Graphs for both areas of interest and each station were created; using these, picking and detection algorithms behavior for each trial can be analyzed.

Figures 5 and 7 show one station results for grid-search and Bayesian optimization. Station DIAM was chosen from among 52 optimized stations in Brazil for having more manual picks. The behavior of all stations is quite similar. 

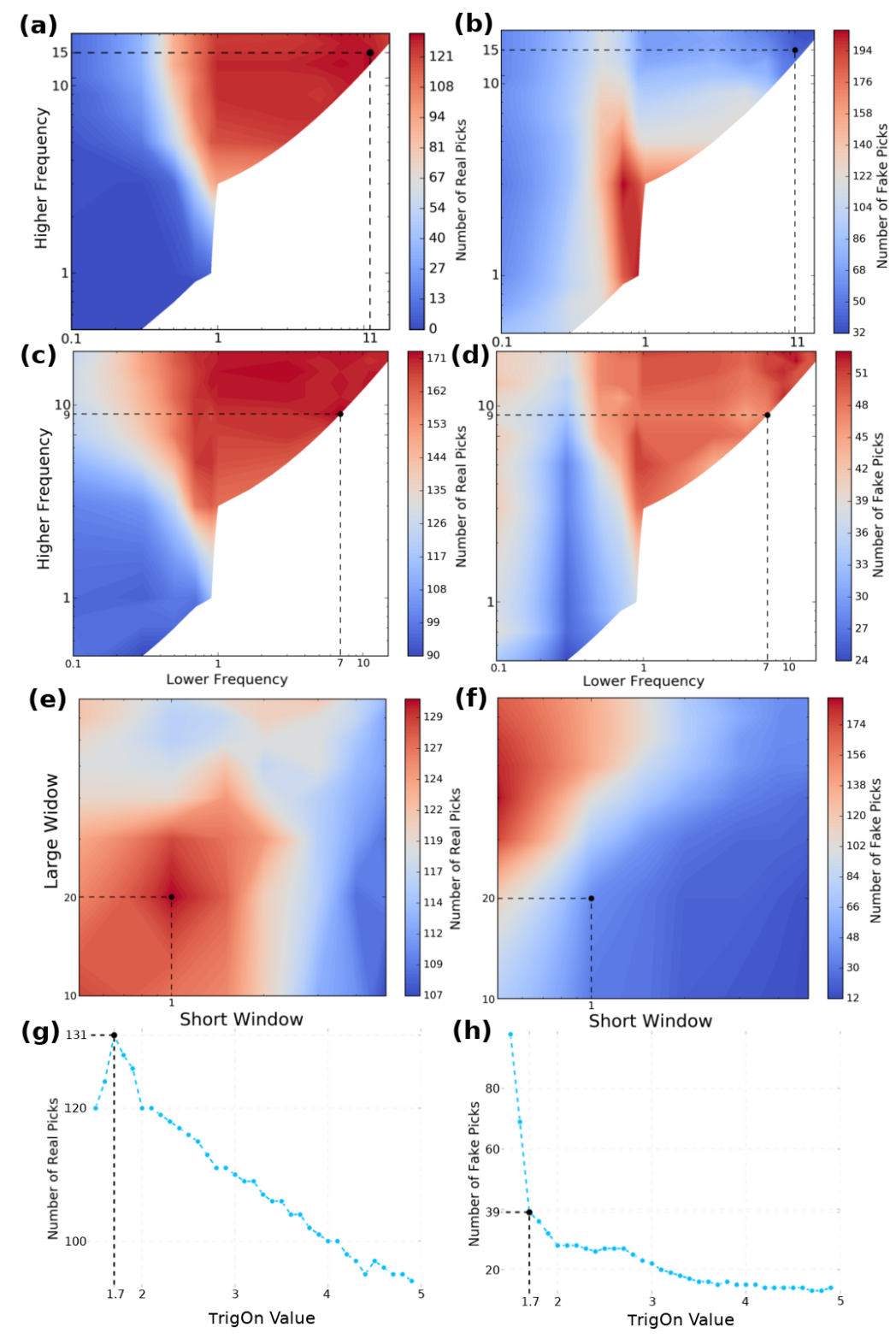

Figure 5: Results for grid-search optimization in DIAM station. Left column with real picks or true positives results and right column with false picks or false positives results. a,b: Corner frequencies for detector's Butherworth. c,d: Corner frequencies for picker's Butherworth. e,f: STA/LTA. g,h: TriggerOn.

The algorithm chooses the best solution by maximizing the number of real picks for all parameters. In figure 5-a, a triangular area with maximum values (red color) is presented. This area was a common feature in the analysis results for almost all stations. Although any trial into this area would produce real picks good results, the code looks for a point where false picks were also reduced. Figure 5 -b, shows an area centered at the point $(0.7,3)$ where the amount of false picks increases considerably; outside of this area, the number of false picks decreases.

Figures 5-c and 5-d show similar areas for real and false picks, a triangular area with maximum values is presented for both graphs. This performance reveals one of the most significant difficulties in doing the optimization process; when we try to maximize real picks, the number of false picks 
increases. Only a few solutions like presented in 5-a and 5-a achieve what we are looking for; increasing real picks and decreases false picks. F1 score was chosen for the Bayesian optimization to find solutions that equilibrates these two requirements.

Solutions for STA/LTA are very narrow for almost all stations. In general, short window values close to one second and large window values between 10 and 30 seconds have good results in increasing real picks and decreasing false ones. See figures 5-e and 5-f.

TrigOn proved to be a critical parameter to optimize. Minor changes in this value produce significant variations in results for real and false picks. Figures 5-g and 5-f shows that using a slightly higher value, the number of real picks decreases considerably, and using a slightly lower value, the number of false picks increases notably. For almost all stations, trigOn values between 1.5 and 2.5 were chosen.

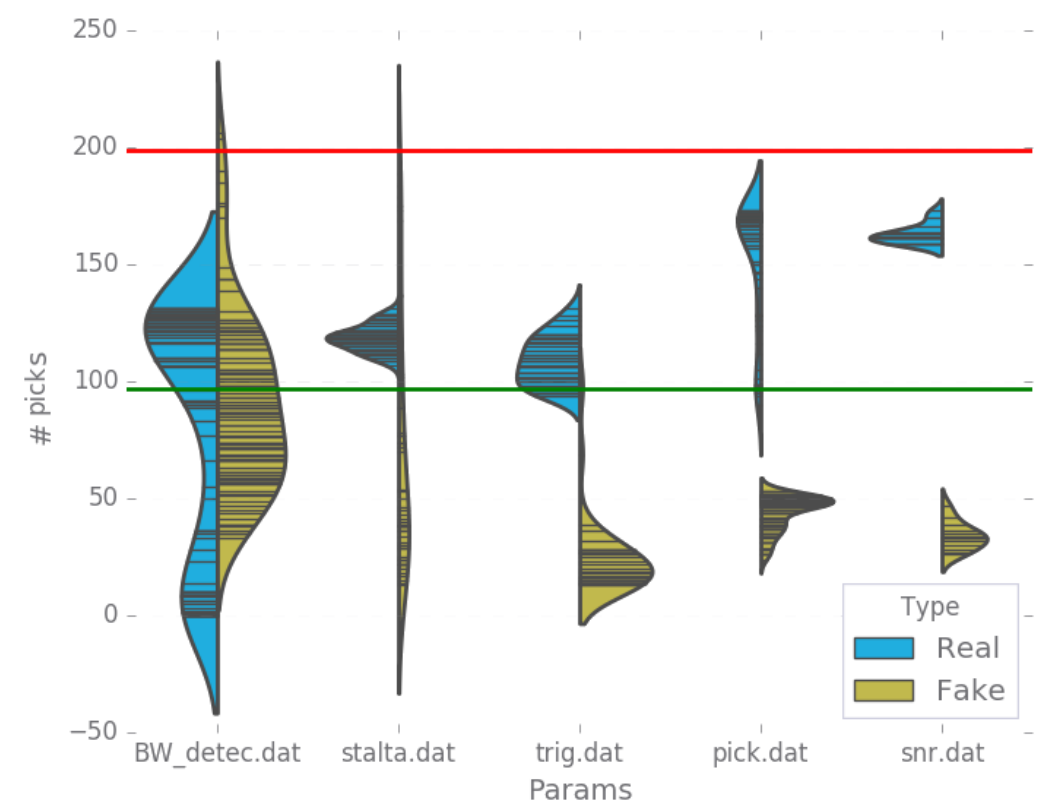

Figure 6: Representation of probability density distribution for the number of picks resulting from all parameter trials. Blue and yellow bars show the distributions of the resulting real and false picks respectively. Green line is the number of real automatic picks before optimization and red line is the number of manual picks.

Figure 6 shows a representation of the probability to obtain some number of real and false picks during the optimization process for each parameter.

It is important to see how the distributions for the first parameter (BW_detect) have a high dispersion, while, for the last optimized parameter (SNR) the dispersion decreases for both real and false picks.

The probability to obtain a low number of real picks decreases after optimizing the detector's Butterworth. On the other hand, the probability to obtain high number of false picks decreases after optimizing STA/LTA. Other essential parameter to optimize is the trigOn which shows high probability to obtain high number of real picks.

It is important to remark how distributions for the last optimized parameter (snr) solutions are concentrated in small areas maximizing real picks and minimizing false picks. The above shows us that although the grid search optimization procedure was done parameter by parameter, using this methodology, the number of picks improved considerably. 

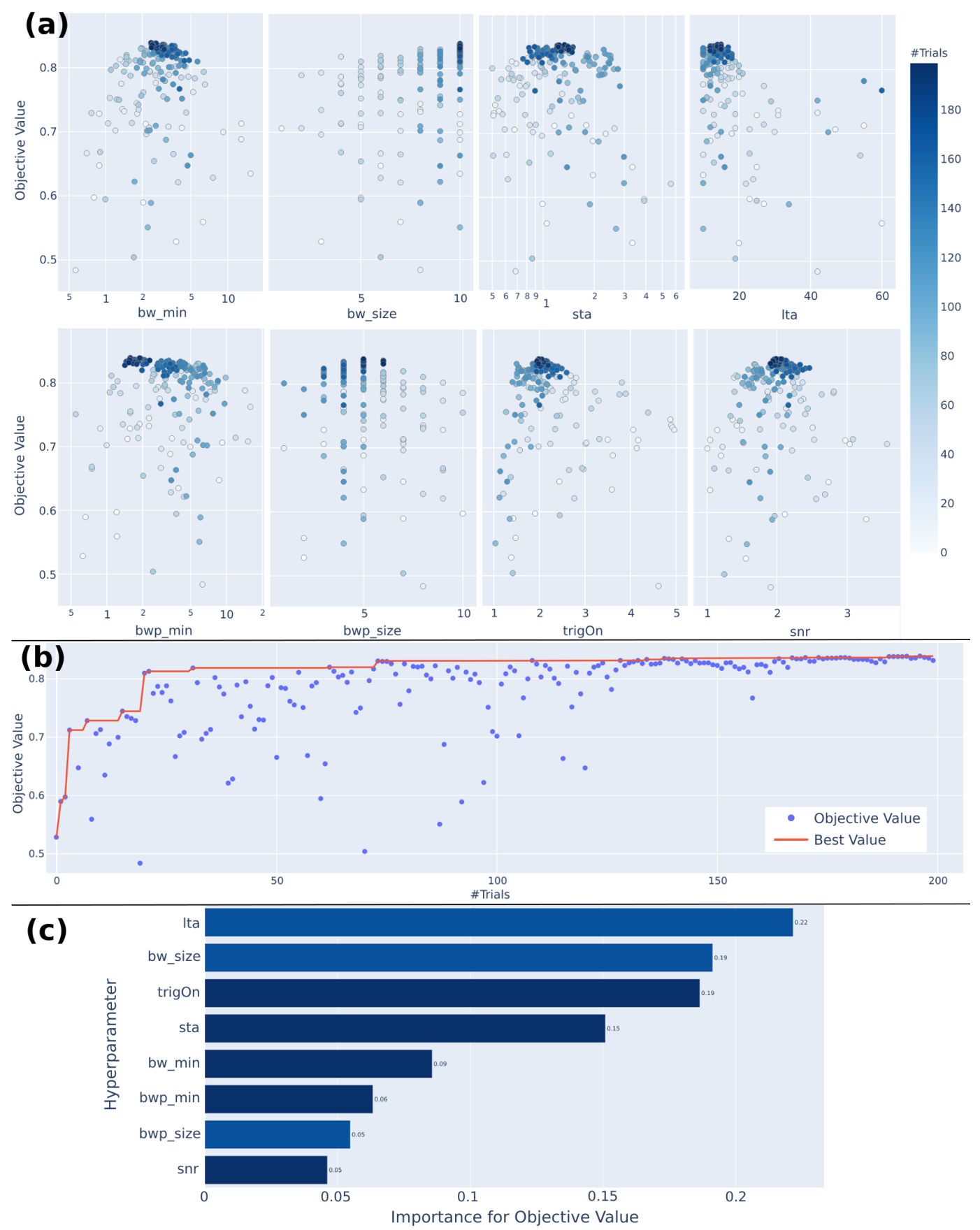

Figure 7: Results for Bayesian optimization in DIAM station. a: Results for each parameters optimized. bw,bwp_min and bw,bwp_size are the lower corner frequencies and filter sizes for detector's and picker's Butherworths. b: Performance of objective value with number of trials. c: Parameter importance.

\section{Bayesian Optimization}

Performance for Bayesian optimization is shown in figure 7 using DIAM station results. The algorithm reaches a well-defined maximum for each of the eight optimized parameters after about 150 results. Solution for detector's Butterworth bw_min and bw_size converges to lower corner frequencies between 2 and $5 \mathrm{~Hz}$ and the size to $10 \mathrm{~Hz}$. These values are similar to the grid-search results, both methodologies produced detector's Butterworth filters with high frequencies up to 15 
Hz. STA results show more dispersion than those for LTA; the best solutions for STA are between 0.7 and $1.5 \mathrm{~s}$ while LTA from 5 to $15 \mathrm{~s}$. Comparing to grid-search results, STA is very similar, and LTA slightly lower for Bayesian results. Picker's Butterworth has the best solutions between 1.5 to $7 \mathrm{~Hz}$ for lower corner frequency and size between 3 to $7 \mathrm{~Hz}$. For trigOn and SNR, there are well-defined maxima close to 2.0 .

The objective value improved steadily until trial 25 when it reached a level close to the optimum; it improved more slowly until stabilizing near trial 150. The above indicates that 200 trials are enough for the algorithm to reach the maximum objective value. See figure 7-B.

Parameter influence in the optimization process was calculated using objective value improvement by varying specific parameter. More critical parameters that increase real picks and decrease false picks are the detector's STA/LTA, trigOn, and Butterworth. This result is similar to what was found using grid-search. See figure 7-C and 5-e.

(a)

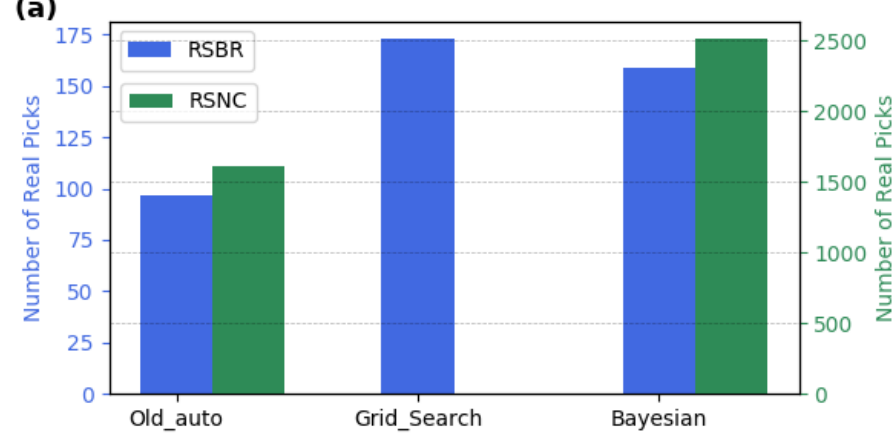

(b)

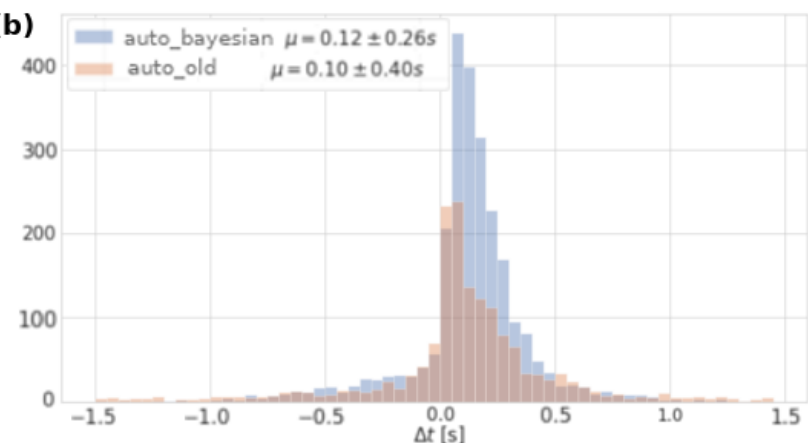

Figure 8: a) Histogram with number of automatic real picks before (Old_auto) and after (Grid_search, Bayesian) optimization process for both seismological centers Brazil RSBR (blue, left axis) and Colombia RSNC (green, right axis). b) Onset time differences between real automatic and manual picks, before (auto_old) and after (auto_bayesian) Bayesian optimization process.

Figure 8-a shows results for automatic picks. For RSNC, the optimization results were tested using a data set different from the data used during the optimization process. Continuous data from July 1 to 14, 2020, were used. Onset times obtained using optimized parameters were compared with those produced using the default configuration of SeisComP. After Bayesian optimization, the automatic processing system identified 2513 picks, $56 \%$ more than using the previous configuration.

On the other hand, for RSBR, we used all available data during the optimization process. For this reason, we test results with the same data set. The automatic processing system identified 173 and 159 picks after grid-search and Bayesian optimization, 78\% and $71 \%$ more, respectively, than using the system configuration before the optimization process.

Automatic pick quality is shown in Figure 8-b. Time differences between manual and automatic picks are similar before and after the optimization process; both means are very close to zero. Most of the comparable picks have a time difference of up to 0.5 seconds.

\section{Discussion}

After the optimization process for the 52 stations part of the RSBR network, using both methodologies, grid-search and Bayesian, we evaluated the results comparing automatic and manual picks. All automatic picks inside a window of 10 seconds around the onset time of manual pick 
were classified as real picks. Other authors have defined this window with similar lengths ((Sleeman and van Eck (1999), Baer and Kradolfer (1987), Leonard (2000) and Chang et al. (2019))), and 8-b shows that onset time differences unusually exceed one second. This methodology is very susceptible to manual pick quality; poor-quality onset time could produce a wrong evaluation of automatic picks.

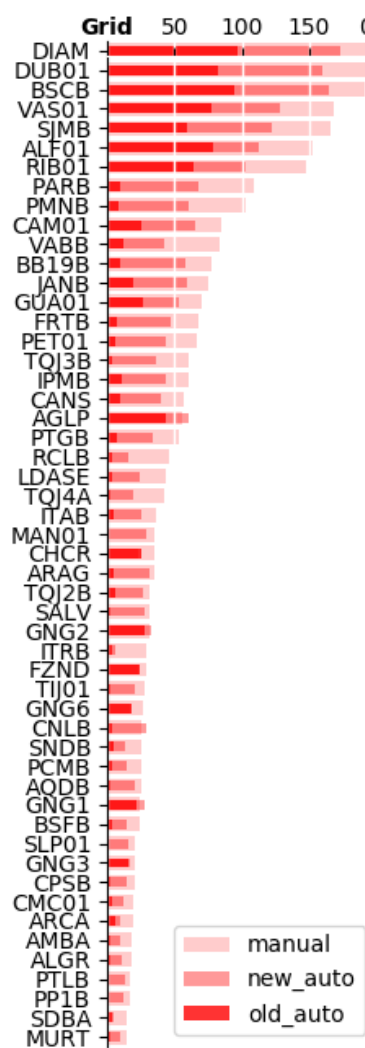

(a)

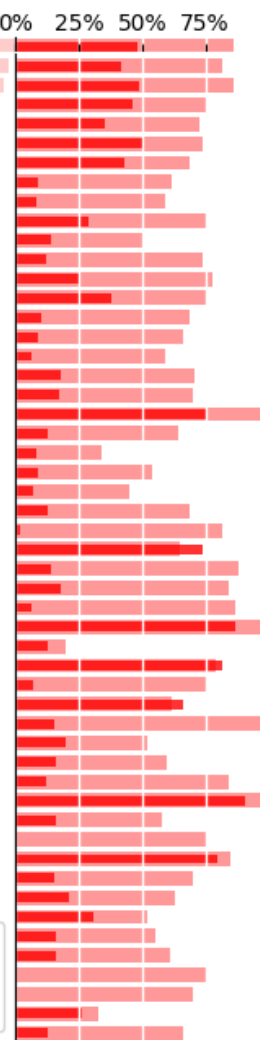

(b)

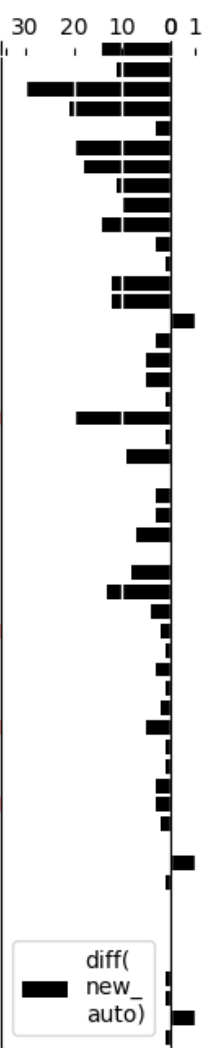

(c)

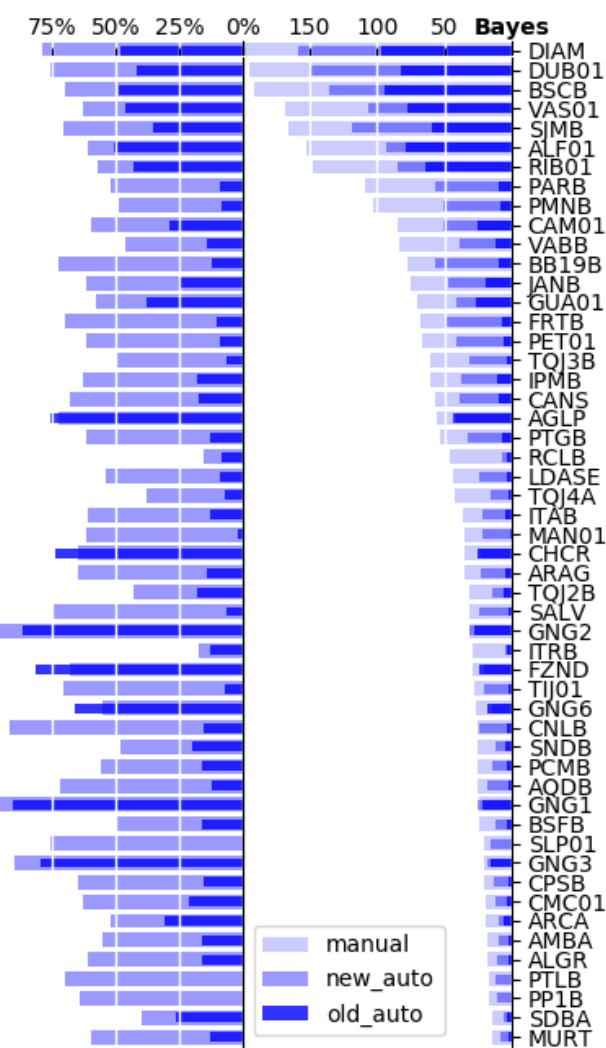

(d)

(e)

Figure 9: Comparison between automatic real picks before (old_auto), after (new_auto) the optimization process and manual picks. Red colors for automatic picks after grid search and blue for Bayesian optimization. -(a): Comparison between old_auto, new_auto and manual picks for all stations, number of picks in the horizontal axis. -(b): Comparison between old_auto and new_auto picks. Percent of automatic picks over manual picks in the horizontal axis. -(c): Difference between new_auto for grid search and new_auto after bayes. If the number of picks is higher for grid search, the difference is plotted to the left; otherwise, it is plotted to the right. -(d) and (e) are the same than (a) and (b) but using results for Bayesian instead of grid search.

Results for real picks are presented in Figure 9. Analyzing histograms figure 9-a and 9-e: -For stations with more than 50 manual picks (above PTGB), the number of real picks after optimization increases and are almost doubled the number of real picks before optimization for both grid and Bayesian methodologies. Only station AGLP has a different result, this station displays more new_auto than manual picks and more old_auto picks than other stations with a similar number of manual picks. This station belongs to a local network that operates in a area of mining activity; therefore, it is a noisy station and could be the reason for the low number of manual picks. To enhance the interpretation we present figure 9-b and 9-d were the percentages of automatic over manual picks are presented. - Only four stations for grid-search and five for Bayesian have less than $50 \%$ picks for new_auto; meanwhile, only seven stations have more than $50 \%$ for old_auto. About $35 \%$ of stations have more than $75 \%$ for new_auto; meanwhile, only 
the $7 \%$ of stations have more than $75 \%$ for old_auto. Some stations like PTLB, PP1B, BSFB, or MAN01 have almost zero real old_auto picks with a considerable improvement after optimization.

The above numbers show us that using either grid search or Bayesian, the performance improves considerably. Improvement with grid search is about $10 \%$ higher than with Bayesian optimization.

The stations AGLP, CHCR, GNG2, FZND, GNG6, GNG1, and GNG3 show a different performance from the others; most of them showed more new_auto picks than previous manual picks. These stations are installed in areas of mining activity and are very noisy. The higher noise level requires a different set of parameters than used for the rest of the network.

Results for real picks show us that using grid-search methodology presents better results than Bayesian; new_auto differences go up to $23 \%$ more picks for BSCB station see figure 9-c. Only three stations have one real pick more for Bayesian than for gird-search, and seven stations have the same number of real picks for both methodologies see figure 9-c.

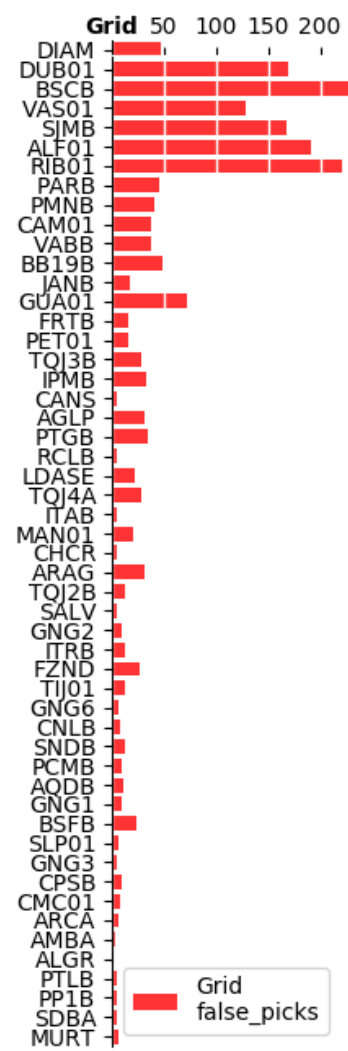

(a)

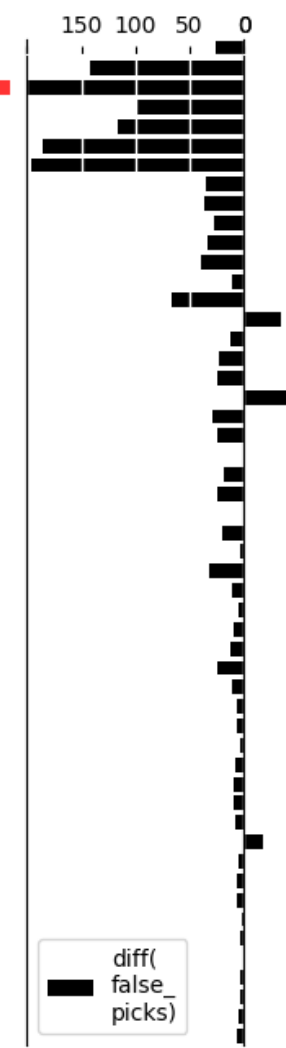

(b)

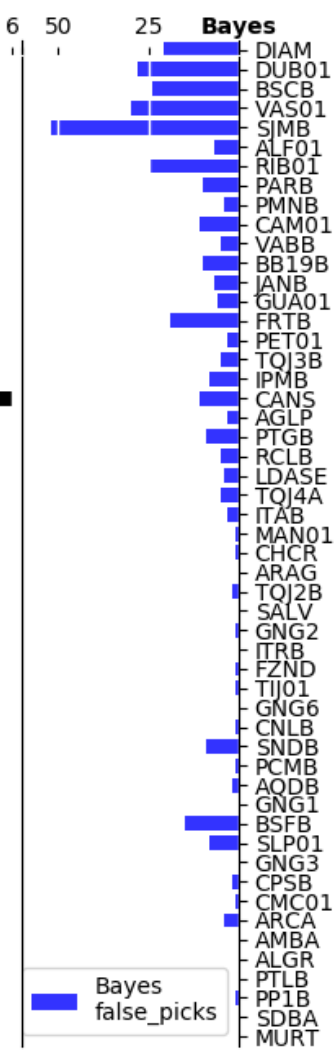

(c)

Figure 10: Comparison between automatic false picks. Red color for automatic picks after grid search and blue for Bayesian optimization. -(a): False picks after grid search optimization process. Number of picks in the horizontal axis. -(b): Difference between false picks for grid search and false picks after Bayesian. If the number of false picks is higher for grid search, the difference is plotted to the left; otherwise, it is plotted to the right. -(c) Is the same than (a) but using results from Bayesian instead of grid search.

On the other hand, results for false picks show better performance for Bayesian methodology than grid-search. Analyzing figure 10-a, stations DUB01, BSCB, VAS01, SJMB, ALF01 and RIB01 have a large number of false picks after grid search optimization. Although these stations after Bayesian optimization have more false picks than other stations see figure 10-c, this methodology present less false picks than grid search. (Figure 10-b). Only three stations (FRTB, CANS and BSFB) have more false picks using Bayesian than grid-search. (Figure 10b). 

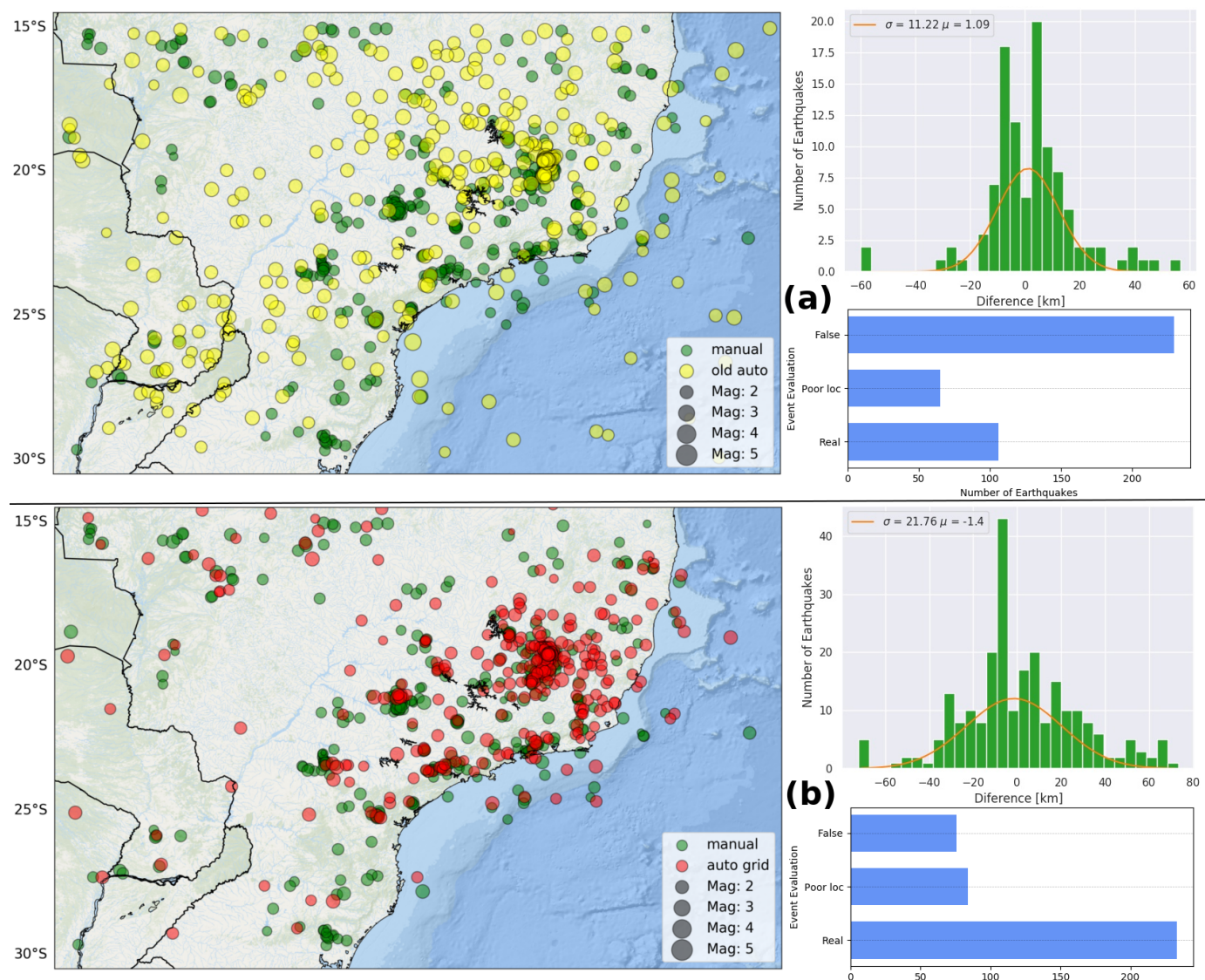

(b)
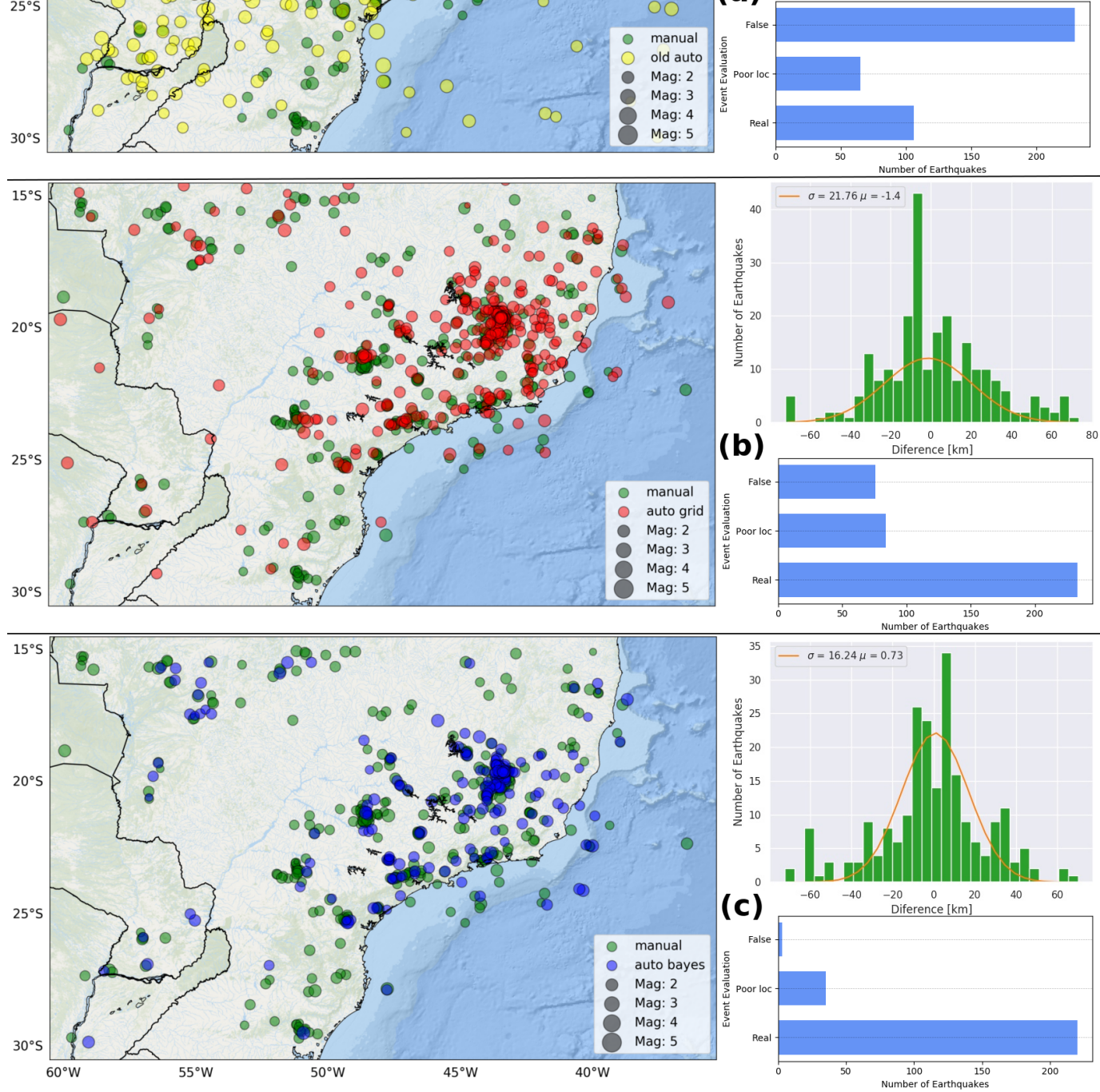

Figure 11: Comparison of automatic versus manual locations. Maps show locations in the interest area next to two histograms; the green one displaying location differences and the blue one with comparison results including false, poor location, and real events. Manual locations versus: -(a): automatic locations before the optimization process. -(b): automatic locations after grid-search optimization. -(c): automatic locations after Bayesian optimization.

Improved pickings were tested on the automatic locations. It is important to emphasize that parameters for location algorithms were not optimized. These locations were calculated using preconfigured automatic location modules for both seismological centers IAG-USP and RSNC. Two SeisComP modules for association picks were used, scautoloc and scanloc (Roessler et al. (2016)). Two location algorithms were used LOCSAT (Bratt and Bache (1988)) and Hypo71. Best results were obtained when using scanloc and Hypo71. 
Manual locations were taken as reference to evaluate automatic locations. We define thresholds of five minutes and $75 \mathrm{~km}$ as the maximum location and time differences to define a pair automaticmanual event as comparable. Both time and distance windows were chosen large enough to include all possible comparable events. A 5 minutes window does not include two different events.

Figure 11 compares manual and automatic locations calculated using picks before and after USP optimization process. Huge dispersion presented in map for old_auto locations (figure 11-a) reduces a lot in comparison with auto_grid and auto_bayes (figure 11-b and c). This dispersion represents false events that decrease from 229 before optimization to 76 and 3 events after optimization using grid-search and Bayesian, respectively. In conclusion, the high number of false events localized before optimization was reduced to one-third after grid-search and practically to zero after Bayesian.

On the other hand, we defined poor-quality localizations for events with differences in origin time less than five minutes but with more than $75 \mathrm{~km}$ in location difference compared to manual events. Poor-quality automatic events increased from 65 to 84 for grid-search and decreased to 35 for Bayesian optimization. (Blue histograms in figure 11).

Finally, a critical result is that real events doubled after optimizations with both grid-search and Bayesian increasing from 106 to 233 and 220, respectively. We can conclude that the results are very satisfactory for both methodologies, but there is a better location quality using Bayesian than grid-search. Grid-search has an rms error of $22 \mathrm{~km}$, Bayesian $16 \mathrm{~km}$. This means that Bayesian picks produce better locations.

A preliminary event localizations were also calculated using the resulting picks after Bayesian optimization for the RSNC. After the optimization process, 153 events were obtained in contrast to the 54 events located automatically before the optimization process. Parameter optimization for grouping picks and location algorithms will be done as part of a new study; therefore, events' location results will be discussed further in future publications.

\section{Conclusions}

- All optimized parameters produced improvements, but some of these show to be more significant than others. Those that offered great changes were the detector's parameters; BW, STA/LTA, and trigOn. This result was obtained using both Grid-search (figure 6) and Bayesian (figure 7-c) optimizations.

- Both methodologies, grid-search maximizing recall and bayesian maximizing f1-score, show excellent results. Similar increases were obtained with these two methodologies for automatic picks (figure 8) and events (11). Grid-search and recall produced the maximum number of comparable automatic picks; using Bayesian and f1-score, computational time and the number of false picks decreases. We choose Bayesian and f1-score as a better methodology because results have better quality by reducing false picks and low-quality events.

- Different types of scores or objective functions can be used according to the optimization purpose. In this work, when using recall, the number of real picks was maximum, but at the same time, the number of false picks was not reduce. Using f1-score a good balance between real and false picks was obtained.

- This work was done for two seismological networks independently to verify the proposed methodology's application for different areas. Results show significant improvements in 
picks and automatic locations after optimization for both networks. This means that the methodologies used in this study are suitable for any seismological network.

- Automatic locations were calculated using the resulting picks after the optimization process. Parameters used in association picks and location algorithms were not optimized; therefore, location results can still improve. Parameter optimization for other algorithms used during automatic processing, such as grouping picks, locator, or origin associator, is necessary to get the most out of the system.

- The trade-off between real and false picks was one of the main difficulties in this optimization process; the balance between these two requirements can only be met using optimization methods next to a suitable objective value. It is unlikely that using manual optimization, an optimal set of parameters can be found.

- Even though not all combinations between parameters were tested in the implementation of grid-search, the results were good. Even though our grid-search used "Recall" as an objective value, the final optimized parameters not only maximized the number of real picks but decreased the number of false picks compared with the old parameters. Additionally, grid-search was helpful to understand and analyze the parameter's performance during the optimization process.

\section{Data and Resources}

Station information, waveforms, and parametric event data used in this study were collected from the Instituto de Astronomia, Geofísica e Ciências Atmosféricas (IAG-USP) in São Paulo University for Brazil and RSNC for Colombia. IAG-USP data can be obtained using the fdsnws service (http://seisarc.sismo.iag.usp.br/fdsnws/) and for Colombia by e-mail request to official accounts shared on the main page (www.sgc.gov.co). All seismological data processing was done using the SeisComP software (https://www.seiscomp.de/). Some routines were developed using ObsPy, a python framework for seismology (https://pypi.org/project/obspy/). Bayesian optimization was implemented using Optuna - A hyperparameter optimization framework (https://optuna.org/). Graphs were done using Matplotlib (https://matplotlib.org/) and maps using Matplotlib Basemap Toolkit (https://matplotlib.org/basemap/).

\section{Acknowledgments}

The authors would like to thank the Instituto de Astronomia, Geofísica e Ciências Atmosféricas and Colombian National Seismological Network for providing the necessary data used in this study. To the seismologist groups from both seismology centers for supporting this research. Work carried out with M.Sc. grant from CAPES. We thank Marcelo Bianchi, Bruno Collaço and Jackson Calhau for implementing the USP SeisComP system to locate events with stations of the RSBR (Brazilian Seismographic Network). José Roberto Barbosa and Cleusa Barbosa manually located all Brazilian events for the USP Seismological Center. 


\section{References}

Akaike, H. (1998). Information Theory and an Extension of the Maximum Likelihood Principle, pages 199-213. Springer New York, New York, NY.

Akiba, T., Sano, S., Yanase, T., Ohta, T., and Koyama, M. (2019). Optuna: A next-generation hyperparameter optimization framework. pages 2623-2631.

Allen, R. (1982). Automatic phase pickers: Their present use and future prospects. Bulletin of the Seismological Society of America, 72(6B):S225-S242.

Allen, R. V. (1978). Automatic earthquake recognition and timing from single traces.

Baer, M. and Kradolfer, U. (1987). An automatic phase picker for local and teleseismic events. Bulletin of the Seismological Society of America, 77(4):1437-1445.

Bergstra, J., Bardenet, R., Bengio, Y., and Kégl, B. (2011a). Algorithms for hyper-parameter optimization. In Proceedings of the 24th International Conference on Neural Information Processing Systems, NIPS'11, page 2546-2554, Red Hook, NY, USA. Curran Associates Inc.

Bergstra, J. S., Bardenet, R., Bengio, Y., and Kégl, B. (2011b). Algorithms for hyper-parameter optimization. In Shawe-Taylor, J., Zemel, R. S., Bartlett, P. L., Pereira, F., and Weinberger, K. Q., editors, Advances in Neural Information Processing Systems 24, pages 2546-2554. Curran Associates, Inc.

Betrò, B. (1992). Bayesian methods in global optimization. In Gritzmann, P., Hettich, R., Horst, R., and Sachs, E., editors, Operations Research '91, pages 16-18, Heidelberg. Physica-Verlag HD.

Bianchi, M. B., Assumpção, M., Rocha, M. P., Carvalho, J. M., Azevedo, P. A., Fontes, S. L., Dias, F. L., Ferreira, J. M., Nascimento, A. F., Ferreira, M. V., and Costa, I. S. L. (2018). The Brazilian Seismographic Network (RSBR): Improving Seismic Monitoring in Brazil. Seismological Research Letters, 89(2A):452-457.

Bratt, S. R. and Bache, T. C. (1988). Locating events with a sparse network of regional arrays. Bulletin of the Seismological Society of America, 78(2):780-798.

Chang, Y.-H., Hung, S.-H., and Chen, Y.-L. (2019). A fast algorithm for automatic phase picker and event location: Application to the 2018 hualien earthquake sequences. Terrestrial, Atmospheric and Oceanic Sciences, 30:435-448.

Chu, C.-K. and Mendel, J. (1994). First break refraction event picking using fuzzy logic systems. IEEE Transactions on Fuzzy Systems, 2(4):255-266.

Colombiano, S. G. (1993). Red sismologica nacional de colombia.

Gentili, S. and Michelini, A. (2006). Automatic picking of p and s phases using a neural tree. Journal of Seismology, 10:39-63.

Géron, A. and Safari, a. O. M. C. (2019). Hands-On Machine Learning with Scikit-Learn, Keras, and TensorFlow, 2nd Edition. O'Reilly Media, Incorporated. 
Hendriyana, A., Bauer, K., Muksin, U., and Weber, M. (2018). AIC-based diffraction stacking for local earthquake locations at the Sumatran Fault (Indonesia). Geophysical Journal International, 213(2):952-962.

Jones, D. (2001). A taxonomy of global optimization methods based on response surfaces. J. of Global Optimization, 21:345-383.

Leonard, M. (2000). Comparison of Manual and Automatic Onset Time Picking. Bulletin of the Seismological Society of America, 90(6):1384-1390.

MAEDA, N. (1985). A method for reading and checking phase time in auto-processing system of seismic wave data. Zisin (Journal of the Seismological Society of Japan. 2nd ser.), 38(3):365379 .

Mockus, J., Tiesis, V., and Zilinskas, A. (2014). The application of Bayesian methods for seeking the extremum, volume 2, pages 117-129.

Olivieri, M., Michelini, A., and Lomax, A. (2007). New robust automatic earthquake locations for the italian region.

Pechmann, J. C. (1998, 2006). Suggested picker parameter changes. Geophysical Research Abstracts, $9(05106)$.

Prieto, G. A., Beroza, G. C., Barrett, S. A., López, G. A., and Florez, M. (2012). Earthquake nests as natural laboratories for the study of intermediate-depth earthquake mechanics. Tectonophysics, 570-571:42-56.

Roessler, D., Becker, J., Ellguth, E., Henneberger, R., Herrnkind, S., and Weber, B. (2016). Cluster-search based monitoring of local earthquakes in seiscomp3.

Saragiotis, C., Hadjileontiadis, L., and Panas, S. (1999). A higher-order statistics-based phase identification ofthree-component seismograms in a redundant wavelet transform domain. pages 396-399.

Sleeman, R. and van Eck, T. (1999). Robust automatic p-phase picking: an on-line implementation in the analysis of broadband seismogram recordings. Physics of the Earth and Planetary Interiors, 113(1):265 - 275 .

Snoek, J., Larochelle, H., and Adams, R. P. (2012). Practical bayesian optimization of machine learning algorithms. In Proceedings of the 25th International Conference on Neural Information Processing Systems - Volume 2, NIPS'12, page 2951-2959, Red Hook, NY, USA. Curran Associates Inc.

Sokolova, M. and Lapalme, G. (2009). A systematic analysis of performance measures for classification tasks. Information Processing y Management, 45(4):427 - 437.

Taijia Xiao, Dong Ren, Shuanghui Lei, Junqiao Zhang, and Xiaobo Liu (2014). Based on gridsearch and pso parameter optimization for support vector machine. In Proceeding of the 11th World Congress on Intelligent Control and Automation, pages 1529-1533. 
Vassallo, M., Satriano, C., and Lomax, A. (2012). Automatic Picker Developments and Optimization: A Strategy for Improving the Performances of Automatic Phase Pickers. Seismological Research Letters, 83(3):541-554.

Weber, B., Becker, J., Hanka, W., Heinloo, A., Hoffmann, M., Kraft, T., Pahlke, D., Reinhardt, J., Saul, J., and Thoms, H. (2007). Seiscomp3 - automatic and interactive real time data processing.

Withers, M., Aster, R., Young, C., Beiriger, J., Harris, M., Moore, S., and Trujillo, J. (1998). A comparison of select trigger algorithms for automated global seismic phase and event detection. Bulletin of the Seismological Society of America, 88:95-106.

Wu, J., Chen, X.-Y., Zhang, H., Xiong, L.-D., Lei, H., and Deng, S.-H. (2019). Hyperparameter optimization for machine learning models based on bayesian optimizationb. Journal of Electronic Science and Technology, 17(1):26 - 40.

Zarifi, Z. and Havskov, J. (2003). Characteristics of dense nests of deep and intermediate-depth seismicity. Advances in Geophysics, 46:237-278.

Zhang, H., Thurber, C., and Rowe, C. (2003). Automatic P-Wave Arrival Detection and Picking with Multiscale Wavelet Analysis for Single-Component Recordings. Bulletin of the Seismological Society of America, 93(5):1904-1912. 


\section{3}

\section{Additional Results}

This chapter includes additional results for different data sets used for the Brazilian Seismic Network (RSBR). The first data set contains all events detected and localized throughout the first semester of 2017 and the second from January/2017 to June/2020. Chapter 2 presented the results using the RSBR data set for January/2017 to June/2020.

The first section shows the second data set using grid-search methodology and recall as the objective value. Several stations were grouped to show results that could help understand the significant variations while optimizing each parameter.

The second section presents the second data set results using Bayesian optimization and f1-score as the objective value. Bayesian methodology results are not as complete as that from grid-search because it does not go through the entire space of possible values for the parameters; nevertheless, some results with special performances are presented in this section.

Finally, the third section shows the first data set results using grid search and recall. Although these results were obtained using a shorter data set, it presents some notable results and a sketch of the grid-search methodology's restrictions for a limited data set. The Bayesian and f1-score methodology was not applied to the first data set. 


\section{ADDITIONAL RESULTS}

\subsection{Grid-search(recall) results for second data set (Jan/2017 - June/2020)}

We now analyze the performance for the detect BW optimization. Figure 3.2 shows a pattern displayed for all stations with a red area representing a high number of false picks. This pattern is present in most of the optimized stations. It is important to emphasize that choosing a BW out of this area only ensures a decrease in the number of false picks for the detection algorithm, the optimization process for other parameters could increase the number of false picks.
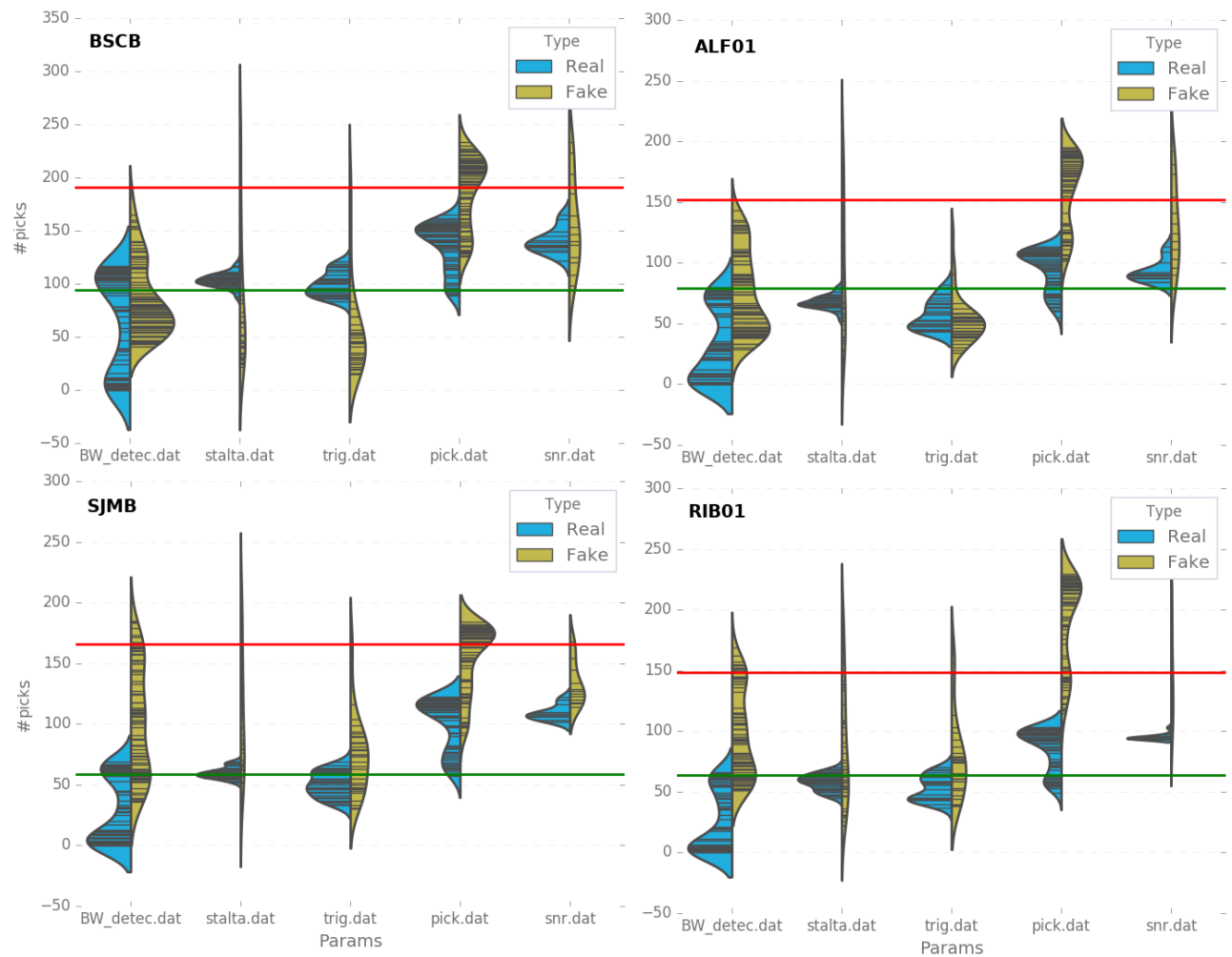

Figure 3.1: Probability density functions for each parameter - Results for four stations are presented. Optimized parameters are presented on $\mathrm{x}$ axis. Blue and yellow colors show the distributions for real and false picks, respectively. The green line is the number of automatic picks before optimization, and the red line is the number of manual picks. 

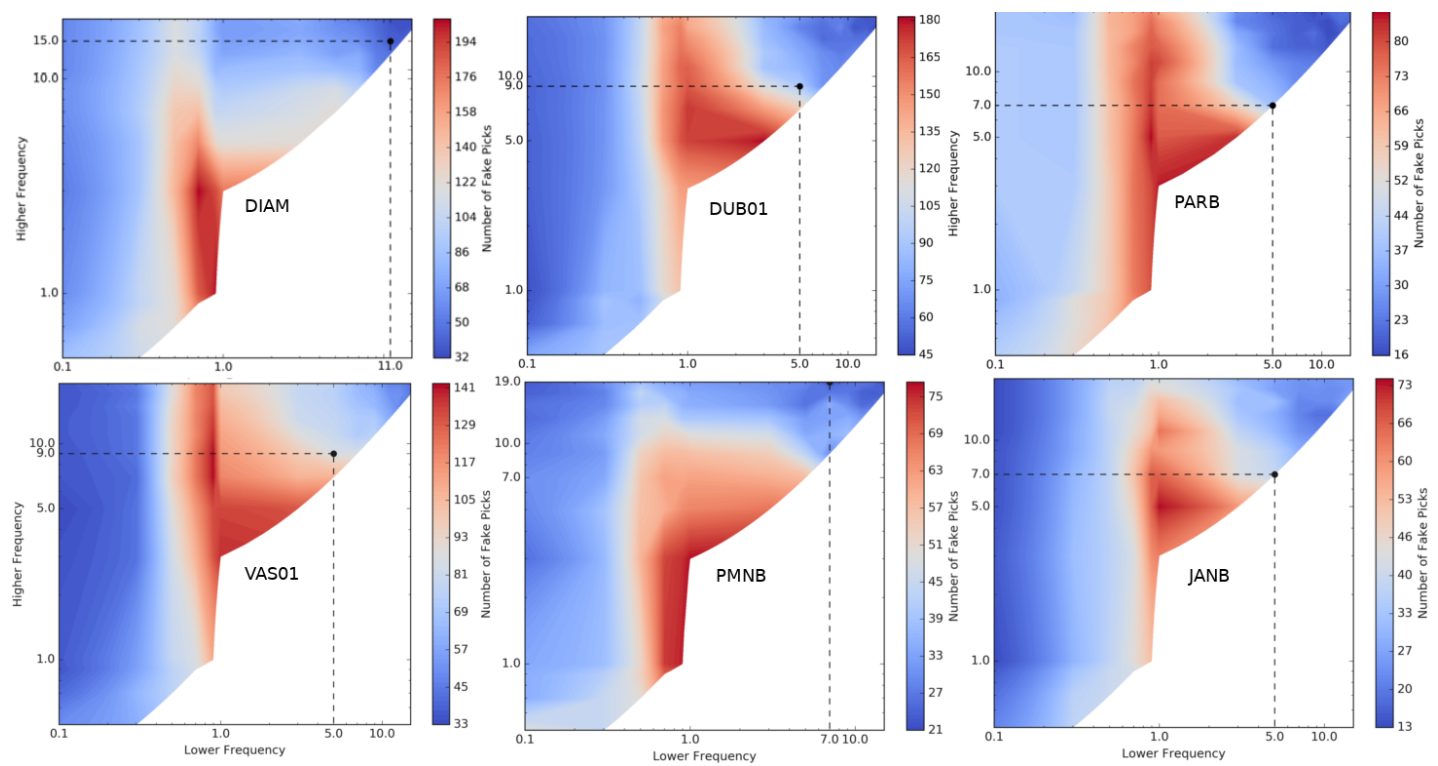

Figure 3.2: False picks results for defect BW optimization - Plots for six stations showing false picks solutions for defect BW lower and higher corner frequencies values. Dashed lines and black mark show the selected solution.

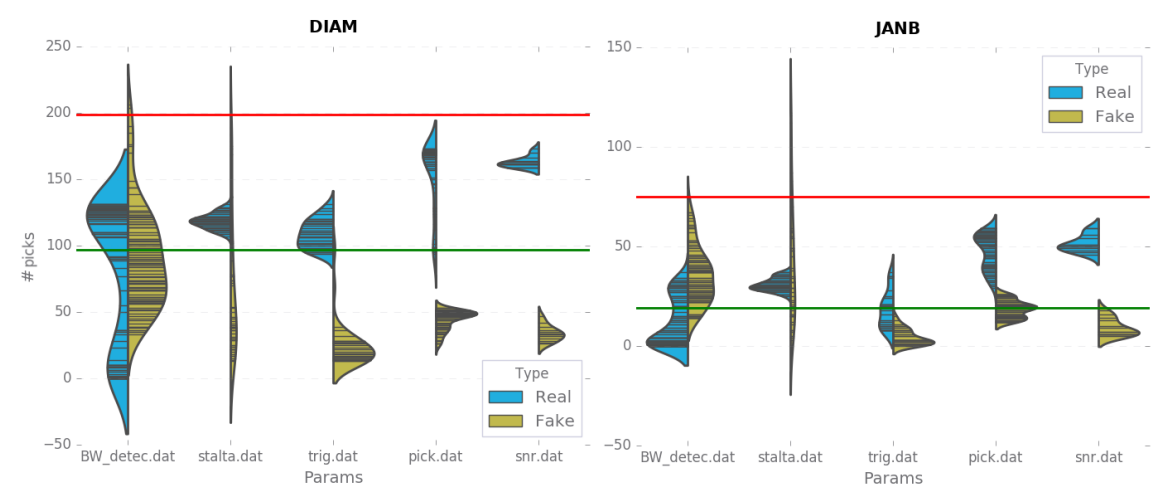

Figure 3.3: Probability density function for each parameter results - Results for two stations are presented. The parameters optimized are presented on $\mathrm{x}$ axis. Blue and yellow colors show the distributions for real and false picks results, respectively. The green line is the number of automatic picks before optimization, and the red line is the number of manual picks.

The resulting probability density function shows the distribution after the optimization process. This tool helps us to understand the performance of each station. One of the expected results is to maximize real picks and minimize false picks. Figure 3.3 


\section{ADDITIONAL RESULTS}

presents these distributions for two stations DIAM and JANB. These stations display high-quality results after the optimization process. Both stations show very concentrated distributions for the "SNR" which was the last parameter optimized. These distributions are very narrow and centered on high values for real picks and low values for false picks. This type of result represents an excellent optimization process. It is important to emphasize that not all stations show this kind of performance; only stations with significant improvements after the optimization process have it.

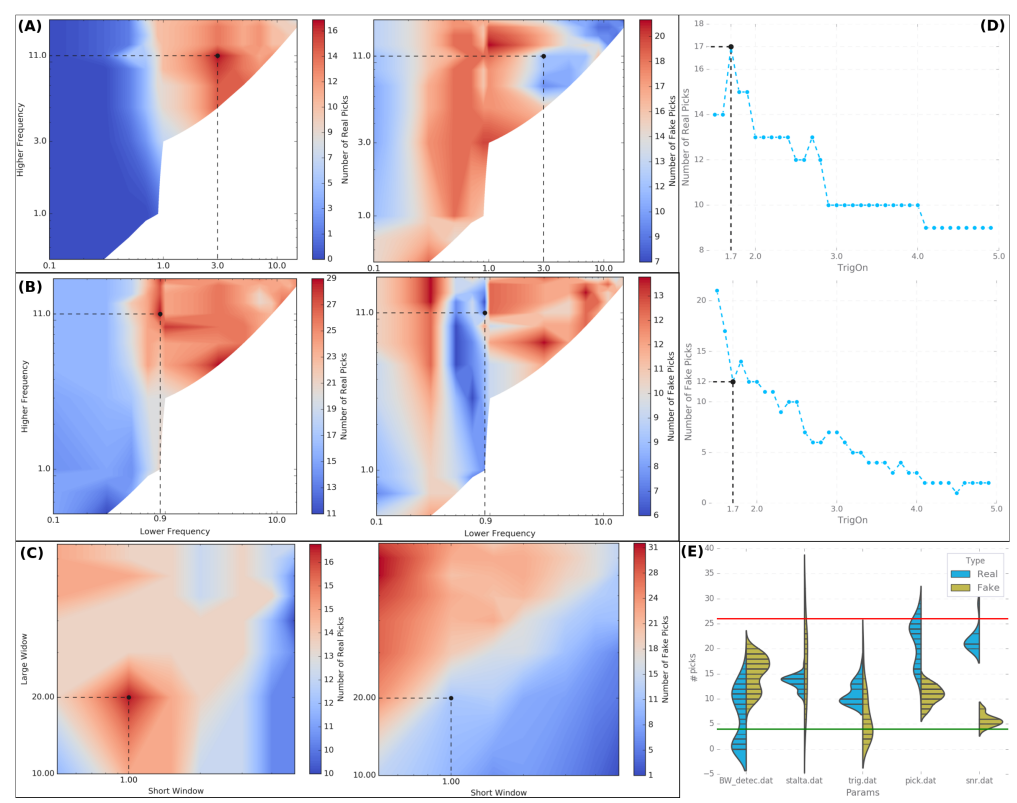

Figure 3.4: False and real picks results for each parameter optimization process in CNLB station. - False an real picks results for: A: Detector BW. B: Picker BW. C: Detector STA/LTA. D: Detector TrigOn. E: Probability density function for each parameter. Blue and yellow bars with the distributions for real and false picks respectively. Green line is the number of automatic picks before optimization and red line is the number of manual picks.

Figure 3.4 presents results for the optimization process in CNLB station. For this station, selected solutions for all parameters agree with red areas for real picks and blue areas for false picks. See figure 3.4 A, B, and C. Figure 3.4 E shows real picks distribution (blue color) centered close to the red line (best result with automatic picks equal to manual picks) for picker's BW and SNR; therefore, the picker's BW is an essential parameter to maximize real picks. This station presents the same performance 
in the results distributions for "SNR" as presented for DIAM and JANB stations in figure 3.3 .

\begin{tabular}{|c|c|c|c|c|c|c|c|c|c|c|c|c|}
\hline & \multicolumn{2}{|c|}{ BW Detector } & \multicolumn{2}{|c|}{ STALTA } & \multicolumn{3}{|c|}{ BW Picker } & \multirow{2}{*}{$\begin{array}{c}\text { SNR } \\
\min \end{array}$} & \multicolumn{4}{|c|}{ Picks } \\
\hline STA & lf & $\mathrm{hf}$ & STA & LTA & trigOn & If & hf & & man & auto & real & false \\
\hline DIAM & 11.0 & 15.0 & 1.0 & 20 & 1.7 & 7.0 & 9.0 & 1.0 & 199 & 97 & 173 & 47 \\
\hline DUB01 & 5.0 & 9.0 & 0.5 & 10 & 1.7 & 5.0 & 17.0 & 1.0 & 195 & 82 & 160 & 170 \\
\hline BSCB & 5.0 & 11.0 & 0.5 & 20 & 1.7 & 15.0 & 19.0 & 1.0 & 191 & 94 & 165 & 233 \\
\hline VAS01 & 5.0 & 9.0 & 1.0 & 20 & 1.7 & 9.0 & 17.0 & 1.0 & 168 & 78 & 128 & 129 \\
\hline SJMB & 5.0 & 9.0 & 1.0 & 20 & 1.7 & 9.0 & 11.0 & 1.0 & 166 & 59 & 122 & 168 \\
\hline ALF01 & 3.0 & 13.0 & 1.0 & 20 & 1.5 & 13.0 & 17.0 & 1.0 & 152 & 79 & 113 & 192 \\
\hline RIB01 & 7.0 & 11.0 & 1.0 & 80 & 1.7 & 3.0 & 13.0 & 1.0 & 148 & 64 & 103 & 221 \\
\hline PARB & 5.0 & 7.0 & 1.0 & 20 & 1.8 & 3.0 & 17.0 & 1.0 & 109 & 10 & 68 & 45 \\
\hline PMNB & 7.0 & 19.0 & 1.0 & 30 & 1.7 & 1.0 & 11.0 & 1.0 & 103 & 9 & 61 & 40 \\
\hline CAM01 & 5.0 & 9.0 & 1.0 & 10 & 1.7 & 3.0 & 19.0 & 1.0 & 85 & 25 & 65 & 38 \\
\hline VABB & 9.0 & 19.0 & 1.0 & 20 & 1.7 & 9.0 & 19.0 & 1.0 & 83 & 12 & 42 & 38 \\
\hline BB19B & 13.0 & 15.0 & 1.0 & 20 & 1.6 & 3.0 & 7.0 & 1.0 & 78 & 10 & 58 & 49 \\
\hline JANB & 5.0 & 7.0 & 2.0 & 10 & 1.7 & 11.0 & 19.0 & 1.2 & 75 & 19 & 59 & 18 \\
\hline GUA01 & 5.0 & 9.0 & 0.5 & 50 & 2.6 & 11.0 & 17.0 & 1.0 & 70 & 27 & 53 & 72 \\
\hline FRTB & 5.0 & 15.0 & 1.0 & 10 & 1.7 & 5.0 & 11.0 & 1.0 & 68 & 7 & 47 & 15 \\
\hline PET01 & 11.0 & 17.0 & 1.0 & 20 & 1.7 & 1.0 & 13.0 & 1.0 & 66 & 6 & 44 & 15 \\
\hline TQJ3B & 5.0 & 9.0 & 1.5 & 40 & 1.7 & 0.5 & 0.9 & 1.0 & 61 & 4 & 36 & 28 \\
\hline IPMB & 3.0 & 9.0 & 1.0 & 20 & 1.7 & 5.0 & 7.0 & 1.2 & 60 & 11 & 43 & 33 \\
\hline CANS & 5.0 & 7.0 & 3.0 & 10 & 1.7 & 0.9 & 19.0 & 1.0 & 57 & 10 & 40 & 5 \\
\hline AGLP & 0.9 & 1.0 & 0.5 & 20 & 2.5 & 0.1 & 11.0 & 1.0 & 56 & 43 & 61 & 32 \\
\hline PTGB & 5.0 & 13.0 & 1.0 & 20 & 1.7 & 13.0 & 17.0 & 1.0 & 53 & 7 & 34 & 34 \\
\hline RCLB & 15.0 & 19.0 & 1.0 & 20 & 1.6 & 5.0 & 13.0 & 1.4 & 46 & 4 & 16 & 5 \\
\hline LDASE & 1.0 & 5.0 & 1.0 & 20 & 1.8 & 0.7 & 0.9 & 1.0 & 44 & 4 & 24 & 22 \\
\hline TQJ4A & 0.7 & 5.0 & 1.0 & 20 & 1.7 & 3.0 & 11.0 & 1.0 & 42 & 3 & 19 & 29 \\
\hline ITAB & 5.0 & 7.0 & 3.0 & 10 & 1.7 & 3.0 & 17.0 & 1.0 & 36 & 5 & 25 & 4 \\
\hline ARAG & 5.0 & 17.0 & 0.5 & 30 & 1.7 & 7.0 & 17.0 & 1.4 & 35 & 5 & 31 & 32 \\
\hline CHCR & 0.7 & 9.0 & 1.0 & 20 & 1.7 & 0.5 & 3.0 & 3.2 & 35 & 26 & 23 & 5 \\
\hline MAN01 & 3.0 & 15.0 & 0.5 & 10 & 1.6 & 11.0 & 15.0 & 1.0 & 35 & 1 & 29 & 21 \\
\hline GNG2 & $\begin{array}{ll}0.7 \\
\end{array}$ & 17.0 & 1.0 & 20 & 1.7 & 5.0 & 15.0 & 1.4 & 32 & 28 & 33 & 10 \\
\hline SALV & 5.0 & 15.0 & 1.0 & 20 & 1.7 & 9.0 & 17.0 & 1.0 & 32 & 2 & 28 & 5 \\
\hline TQJ2B & 11.0 & 13.0 & 1.0 & 30 & 1.7 & 0.3 & 3.0 & 1.0 & 32 & 6 & 27 & 13 \\
\hline FZND & 0.9 & 1.0 & 0.5 & 50 & 1.7 & 0.9 & 1.0 & 3.4 & 29 & 24 & 23 & 26 \\
\hline ITRB & 7.0 & 11.0 & 1.0 & 20 & 1.7 & 0.5 & 7.0 & 1.0 & 29 & 4 & 6 & 12 \\
\hline TIJ01 & 1.0 & 15.0 & 1.0 & 20 & 1.7 & 5.0 & 17.0 & 1.0 & 28 & 2 & 21 & 12 \\
\hline GNG6 & 0.9 & 9.0 & 1.0 & 20 & 1.7 & 1.0 & 3.0 & 1.0 & 27 & 18 & 17 & 6 \\
\hline CNLB & 3.0 & 11.0 & 1.0 & 20 & 1.7 & 0.9 & 11.0 & 1.0 & 26 & 4 & 29 & 8 \\
\hline AQDB & 5.0 & 7.0 & 1.0 & 20 & 1.7 & 15.0 & 19.0 & 1.0 & 25 & 3 & 21 & 11 \\
\hline PCMB & 7.0 & 11.0 & 4.0 & 70 & 1.7 & 9.0 & 17.0 & 1.2 & 25 & 4 & 15 & 9 \\
\hline SNDB & 9.0 & 11.0 & 1.0 & 10 & 1.7 & 0.3 & 17.0 & 1.0 & 25 & 5 & 13 & 12 \\
\hline BSFB & 5.0 & 9.0 & 1.0 & 20 & 1.7 & 9.0 & 15.0 & 1.2 & 24 & 4 & 14 & 23 \\
\hline GNG1 & 0.7 & 5.0 & 1.0 & 20 & 1.8 & 0.5 & 3.0 & 1.4 & 24 & 22 & 28 & 10 \\
\hline SLP01 & 3.0 & 7.0 & 1.0 & 10 & 1.8 & 5.0 & 17.0 & 1.0 & 21 & 0 & 16 & 6 \\
\hline CPSB & 3.0 & 11.0 & 1.0 & 10 & 1.7 & 0.9 & 13.0 & 1.4 & 20 & 3 & 14 & 9 \\
\hline GNG3 & 0.9 & 15.0 & 1.5 & 20 & 1.7 & 0.9 & 5.0 & 1.0 & 20 & 16 & 17 & 5 \\
\hline ARCA & 3.0 & 5.0 & 1.0 & 10 & 1.7 & 1.0 & 3.0 & 1.0 & 19 & 6 & 10 & 6 \\
\hline CMC01 & 7.0 & 9.0 & 1.0 & 10 & 1.7 & 3.0 & 5.0 & 1.6 & 19 & 4 & 12 & 8 \\
\hline ALGR & 7.0 & 9.0 & 1.0 & 20 & 2.8 & 0.9 & 9.0 & 1.0 & 18 & 3 & 11 & 0 \\
\hline AMBA & 7.0 & 17.0 & 1.0 & 20 & 1.7 & 7.0 & 9.0 & 1.2 & 18 & 3 & 10 & 3 \\
\hline PP1B & 13.0 & 17.0 & 1.0 & 20 & 1.7 & 15.0 & 19.0 & 1.2 & 17 & 0 & 12 & 5 \\
\hline PTLB & 11.0 & 17.0 & 1.0 & 20 & 1.7 & 1.0 & 3.0 & 1.0 & 17 & 0 & 13 & 4 \\
\hline MURT & 5.0 & 9.0 & 0.5 & 10 & 1.8 & 5.0 & 7.0 & 1.0 & 15 & 2 & 10 & 6 \\
\hline SDBA & 3.0 & 7.0 & 1.0 & 10 & 1.7 & 7.0 & 17.0 & 1.0 & 15 & 4 & 5 & 5 \\
\hline
\end{tabular}

Table 3.1: Table with the results using Grid-search(recall) optimization for second data set (Jan/2017 - June/2020). BW: Butterworth. lf: Low frequency. hf: High frequency. man: Manual picks. auto: Automatic picks before optimization. real: Automatic picks after optimization. False: False automatic picks after optimization. 


\subsection{Bayesian(f1-score) results for second data set (Jan/2017 - June/2020)}

From all stations with more than 100 manual picks, SJMB is the station with the lowest difference of real picks between both optimization methodologies used in this study. See figure 9 in the paper. Furthermore, this station has the highest number of false picks from all stations optimized with Bayesian and f1-score. In figure 3.5 we present results for the optimization process for the SJMB station. This station shows three principal differences compared to other stations performance. First, the objective function did not converge to the maximum value close to 0.7 but fluctuate from 0.6 up to 0.7 . Second, the selected picker BW goes up to $17 \mathrm{~Hz}$, generating more false picks than expected. Finally, performance for the SNR optimization shows two maxima for the objective value; indeed, the maximum close to 3.5 , which was not selected by the code, could produce fewer false picks, but fewer real picks too.

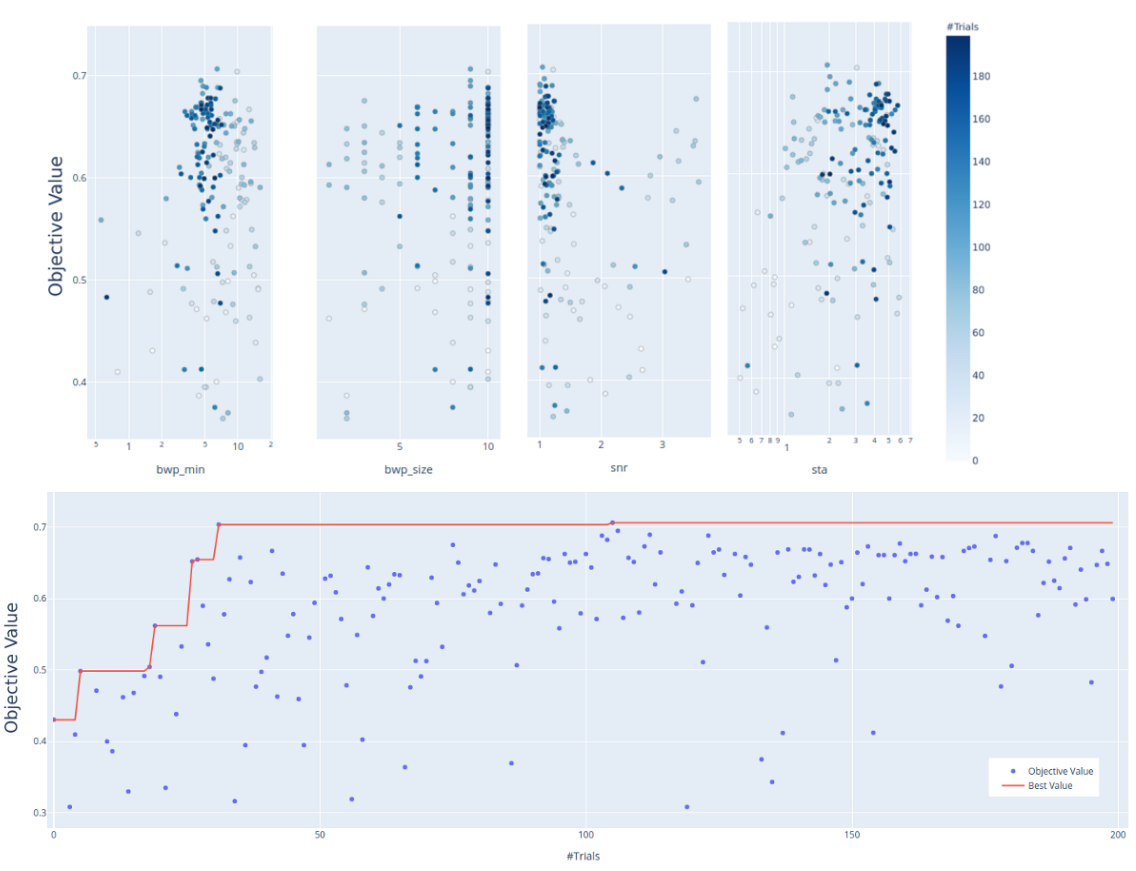

Figure 3.5: Bayesian and f1-score optimization results for SJMB station - Four graphs at the top show optimization results for Picker's BW, SNR, and STA parameters. Lower corner frequency represented with bwp_min and size filter with bwp_size. The figure at the bottom shows the evolution of the objective value with the number of trials. 
Some stations such as AGLP, CHCR, and FZND have an unusually high number of automatic picks before the optimization process and even more than automatic picks after the optimization. This unusual performance could be explained using SNR optimization results for AGLP station. Figure 3.6 presents two possible values for SNR, one close to 2 and the other close to 3.5. The code selected the highest value, which is slightly higher than usual; if the station is very noisy, this value could be selected for producing fewer real picks and fewer false picks, resulting in an f1-score maximum.

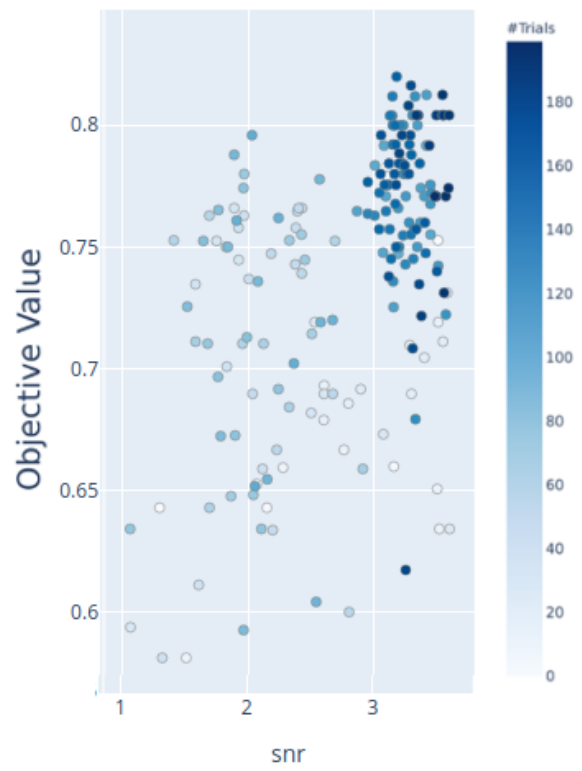

Figure 3.6: SNR optimization results for AGLP station. - Figure with objective function results for SNR values tested in the Bayesian and f1-score optimization.

BSCB station showed the largest difference (for real and false picks) between the two methods. Figures 9 and 10 (in the paper) show 30 more real picks and 200 more false picks using grid-search and recall instead of Bayesian and f1-score. Figure 3.7 shows optimization results for BSCB station and exhibits good convergence for objective values maximums for all parameters optimized and the number of trials. These results confirm what was expected and is summarized in the next three statements:

1. Using Bayesian methodology solution converges with less number of trials than using exhaustive testing with grid-search.

2. Using f1-score instead of recall, the number of false picks decreases significantly. 
3. Using f1-score instead of recall the number of real picks decrease.

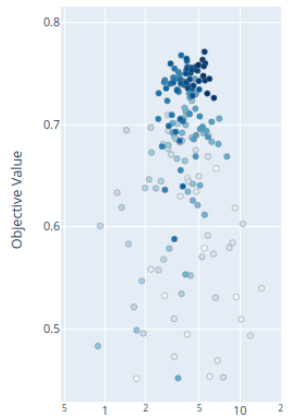

bw_min

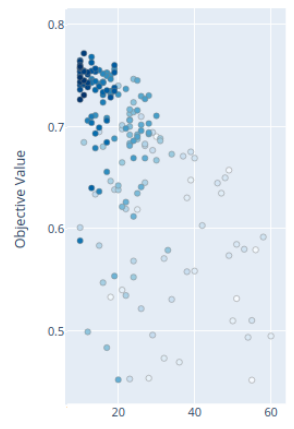

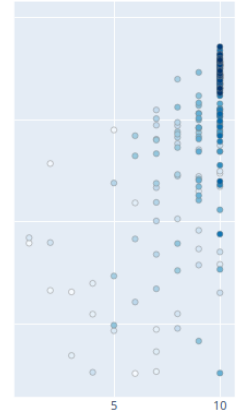

bw_size

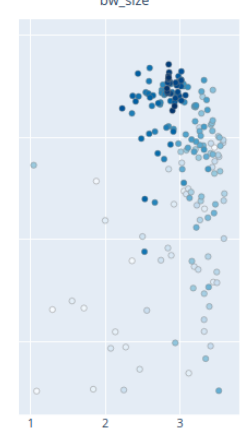

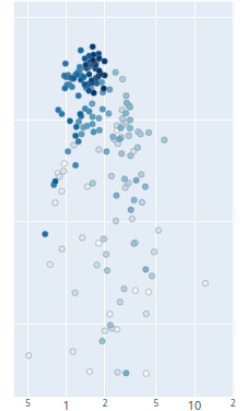

bwp_min

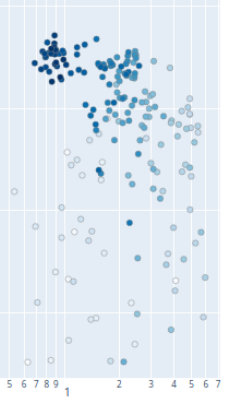

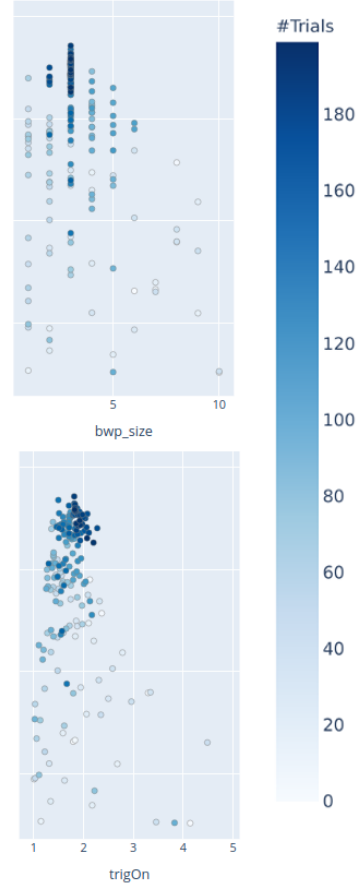

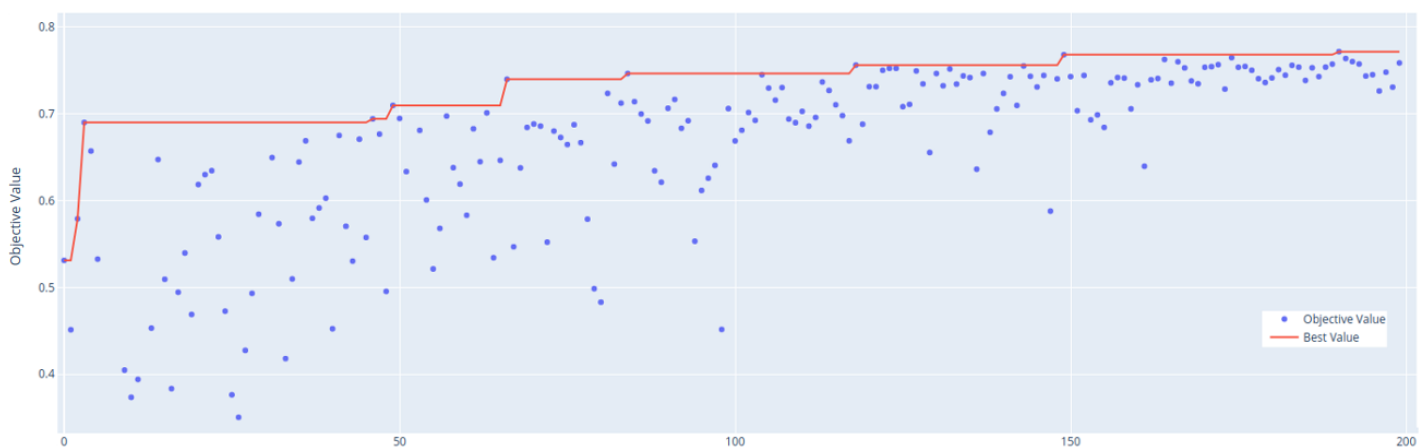

Figure 3.7: Bayesian and f1-score optimization results for BSCB station - Eight graphs at the top show optimization results for detector's BW, picker's BW, STA/LTA, SNR, and trigOn parameters. BW lower corner frequencies represented with bwp_min and bw_min, sizes of BW filters with bwp_size and bw_size. The figure at the bottom shows the performance of the objective value with the number of trials.

Not all stations produced good quality results after Bayesian and f1-score optimization process. Stations such as RCLB and ITRB present the lowest numbers of real picks after optimization. See figures 9 and 10 (in the paper). Figure 3.8 shows objective value evolution with the number of trials for RCLB station; the maximum objective value 
close to 0.25 is quite low compared to other stations' results. It is essential to emphasize that improvements were produced when using grid-search and recall optimization for this station. Thus, this station represents an example of poor results using Bayesian and f1-score methodology.

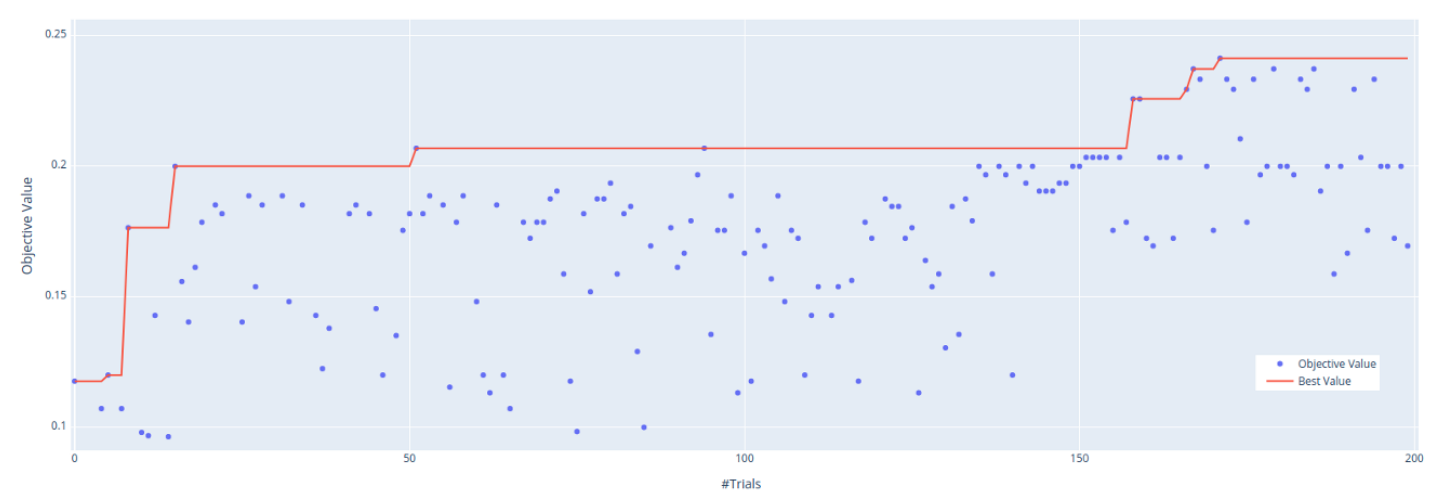

Figure 3.8: Objective value results for RCLB station - Evolution of the objective value with the number of trials for RCLB station. 


\section{ADDITIONAL RESULTS}

\begin{tabular}{|c|c|c|c|c|c|c|c|c|c|c|c|c|}
\hline & \multicolumn{2}{|c|}{ BW Detector } & \multicolumn{2}{|c|}{ STALTA } & \multicolumn{3}{|c|}{ BW Picker } & \multirow{2}{*}{$\frac{\text { SNR }}{\min }$} & \multicolumn{4}{|c|}{ Picks } \\
\hline STA & lf & $\mathrm{hf}$ & STA & LTA & trigOn & lf & hf & & $\operatorname{man}$ & auto & real & false \\
\hline DIAM & 2.4 & 12.4 & 1 & 15 & 2.0 & 1.6 & 6.6 & 2.0 & 199 & 97 & 159 & 21 \\
\hline DUB01 & 5.4 & 12.4 & 3 & 12 & 1.3 & 5.0 & 13.0 & 1.6 & 195 & 82 & 149 & 28 \\
\hline $\mathrm{BSCB}$ & 5.5 & 15.5 & 0.9 & 11 & 1.8 & 1.6 & 4.6 & 2.9 & 191 & 94 & 135 & 24 \\
\hline VAS01 & 6.6 & 16.6 & 1 & 11 & 1.6 & 2.2 & 9.2 & 2.8 & 168 & 78 & 107 & 30 \\
\hline SJMB & 2.6 & 11.6 & 2 & 10 & 1.7 & 6.4 & 15.4 & 1.0 & 166 & 59 & 119 & 52 \\
\hline ALF01 & 7.6 & 10.6 & 0.9 & 12 & 1.9 & 6.7 & 10.7 & 2.7 & 152 & 79 & 93 & 7 \\
\hline RIB01 & 5.7 & 11.7 & 2 & 15 & 1.7 & 2.4 & 8.4 & 1.9 & 148 & 64 & 85 & 25 \\
\hline PARB & 9.6 & 13.6 & 1 & 14 & 1.6 & 14.1 & 22.1 & 2.6 & 109 & 10 & 57 & 10 \\
\hline PMNB & 6.4 & 14.4 & 2 & 23 & 1.5 & 1.5 & 11.5 & 2.7 & 103 & 9 & 51 & 4 \\
\hline CAM01 & 5.2 & 15.2 & 0.6 & 10 & 2.5 & 8.1 & 9.1 & 1.4 & 85 & 25 & 51 & 11 \\
\hline VABB & 9.2 & 19.2 & 0.7 & 14 & 1.9 & 10.6 & 20.6 & 3.3 & 83 & 12 & 39 & 5 \\
\hline BB19B & 9.6 & 10.6 & 0.5 & 15 & 2.2 & 6.2 & 15.2 & 1.9 & 78 & 10 & 57 & 10 \\
\hline JANB & 4.1 & 9.1 & 2 & 12 & 1.4 & 2.8 & 11.8 & 2.7 & 75 & 19 & 47 & 7 \\
\hline GUA01 & 4.0 & 11.0 & 3 & 10 & 1.9 & 15.9 & 22.9 & 1.1 & 70 & 27 & 41 & 6 \\
\hline FRTB & 5.4 & 13.4 & 0.8 & 13 & 1.8 & 8.0 & 9.0 & 1.2 & 68 & 7 & 48 & 19 \\
\hline PET01 & 15.9 & 21.9 & 1.0 & 14 & 1.5 & 8.9 & 18.9 & 3.0 & 66 & 6 & 41 & 3 \\
\hline TQJ3B & 5.3 & 6.3 & 1 & 10 & 2.0 & 2.5 & 8.5 & 3.6 & 61 & 4 & 31 & 5 \\
\hline IPMB & 4.6 & 12.6 & 1 & 11 & 1.6 & 6.1 & 7.1 & 1.4 & 60 & 11 & 38 & 8 \\
\hline CANS & 3.4 & 7.4 & 0.7 & 12 & 2.8 & 12.6 & 16.6 & 1.1 & 57 & 10 & 39 & 11 \\
\hline AGLP & 0.5 & 4.5 & 0.6 & 16 & 1.7 & 2.2 & 10.2 & 3.2 & 56 & 43 & 41 & 3 \\
\hline PTGB & 8.3 & 10.3 & 0.9 & 10 & 1.9 & 11.6 & 17.6 & 1.3 & 53 & 7 & 33 & 9 \\
\hline RCLB & 0.9 & 8.9 & 0.8 & 50 & 2.2 & 0.6 & 9.6 & 2.4 & 46 & 4 & 7 & 5 \\
\hline LDASE & 1.6 & 5.6 & 0.6 & 12 & 2.0 & 4.3 & 8.3 & 3.3 & 44 & 4 & 24 & 4 \\
\hline TQJ4A & 1.3 & 3.3 & 3 & 12 & 1.7 & 6.5 & 7.5 & 2.6 & 42 & 3 & 16 & 5 \\
\hline ITAB & 2.9 & 11.9 & 2 & 12 & 1.6 & 5.5 & 9.5 & 2.4 & 36 & 5 & 22 & 3 \\
\hline ARAG & 14.5 & 15.5 & 0.8 & 22 & 2.2 & 10.7 & 19.7 & 3.0 & 35 & 5 & 23 & 0 \\
\hline $\mathrm{CHCR}$ & 3.9 & 11.9 & 1.0 & 27 & 2.5 & 0.8 & 1.8 & 1.3 & 35 & 26 & 23 & 1 \\
\hline MAN01 & 5.5 & 15.5 & 4 & 15 & 1.3 & 16.0 & 19.0 & 2.7 & 35 & 1 & 22 & 1 \\
\hline GNG2 & 0.8 & 8.8 & 1 & 26 & 2.0 & 1.6 & 7.6 & 3.5 & 32 & 28 & 31 & 1 \\
\hline SALV & 8.2 & 18.2 & 1 & 38 & 1.8 & 8.1 & 9.1 & 2.5 & 32 & 2 & 24 & 0 \\
\hline TQJ2B & 1.0 & 10.0 & 0.7 & 14 & 1.7 & 3.7 & 13.7 & 3.0 & 32 & 6 & 14 & 2 \\
\hline FZND & 0.6 & 1.6 & 1 & 33 & 2.2 & 0.6 & 1.6 & 2.1 & 29 & 24 & 20 & 1 \\
\hline ITRB & 2.0 & 10.0 & 3 & 20 & 1.5 & 14.7 & 23.7 & 3.0 & 29 & 4 & 5 & 0 \\
\hline TIJ01 & 9.3 & 18.3 & 0.7 & 55 & 2.9 & 13.5 & 20.5 & 1.0 & 28 & 2 & 20 & 1 \\
\hline GNG6 & 12.9 & 21.9 & 6 & 27 & 1.6 & 14.2 & 24.2 & 3.4 & 27 & 18 & 15 & 0 \\
\hline CNLB & 4.3 & 6.3 & 0.9 & 12 & 1.8 & 4.5 & 5.5 & 2.7 & 26 & 4 & 24 & 1 \\
\hline AQDB & 5.4 & 10.4 & 0.9 & 29 & 1.9 & 4.7 & 9.7 & 2.7 & 25 & 3 & 18 & 2 \\
\hline PCMB & 5.8 & 12.8 & 0.5 & 19 & 2.0 & 6.7 & 15.7 & 3.6 & 25 & 4 & 14 & 1 \\
\hline SNDB & 1.5 & 10.5 & 0.7 & 10 & 2.0 & 0.5 & 3.5 & 1.1 & 25 & 5 & 12 & 9 \\
\hline BSFB & 3.4 & 12.4 & 0.7 & 11 & 1.6 & 7.5 & 17.5 & 3.0 & 24 & 4 & 12 & 15 \\
\hline GNG1 & 4.4 & 10.4 & 2 & 18 & 1.7 & 0.8 & 3.8 & 2.5 & 24 & 22 & 25 & 0 \\
\hline SLP01 & 4.8 & 14.8 & 0.8 & 59 & 1.9 & 16.0 & 22.0 & 1.3 & 21 & 0 & 16 & 8 \\
\hline CPSB & 6.0 & 11.0 & 1 & 25 & 1.9 & 5.5 & 10.5 & 3.4 & 20 & 3 & 13 & 2 \\
\hline GNG3 & 0.8 & 2.8 & 1 & 24 & 2.5 & 1.4 & 6.4 & 2.6 & 20 & 16 & 18 & 0 \\
\hline ARCA & 3.3 & 7.3 & 1 & 10 & 2.6 & 0.6 & 9.6 & 1.1 & 19 & 6 & 10 & 4 \\
\hline $\mathrm{CMC01}$ & 5.4 & 7.4 & 2 & 13 & 2.2 & 3.6 & 12.6 & 1.3 & 19 & 4 & 12 & 1 \\
\hline ALGR & 3.1 & 6.1 & 1.0 & 13 & 2.6 & 4.6 & 10.6 & 1.4 & 18 & 3 & 11 & 0 \\
\hline AMBA & 7.0 & 14.0 & 1 & 13 & 1.6 & 3.9 & 13.9 & 2.7 & 18 & 3 & 10 & 0 \\
\hline PP1B & 14.0 & 17.0 & 1.0 & 22 & 2.0 & 0.6 & 9.6 & 1.8 & 17 & 0 & 11 & 1 \\
\hline PTLB & 7.1 & 11.1 & 0.8 & 18 & 2.6 & 0.6 & 8.6 & 2.5 & 17 & 0 & 12 & 0 \\
\hline MURT & 10.0 & 16.0 & 2 & 21 & 1.9 & 4.8 & 7.8 & 2.9 & 15 & 2 & 9 & 0 \\
\hline SDBA & 4.5 & 11.5 & 1.0 & 27 & 1.8 & 5.7 & 13.7 & 3.0 & 15 & 4 & 6 & 0 \\
\hline
\end{tabular}

Table 3.2: Table with the results using Bayesian(f1-score) optimization for second data set (Jan/2017 - June/2020). BW: Butterworth. lf: Low frequency. hf: High frequency. man: Manual picks. auto: Automatic picks before optimization. real: Automatic picks after optimization. False: False automatic picks after optimization. 


\subsection{Grid-search(recall) results for first data set (Jan to July /2017)}

\subsubsection{Good performance in real automatic picks}

Stations with the best performance after optimization process.

More than $75 \%$ of manual picks produced automatically:

DUB01, ALF01, DIAM, BSCB, SJMB, PMNB, GUA01, JANB, IPMB, AQDB,

CRSM, TQJ2B, FRTB, PCMB

Within this group, the stations that stand out are: DUB01, PMNB, GUA01, JANB, IPMB, AQDB, TQJ2B, FRTB, PCMB

These stations improve more than $50 \%$ in real picks.

Following, we present graphs showing the result after parameter optimization for the station DUB01. This station was chosen to represent all stations from this group:

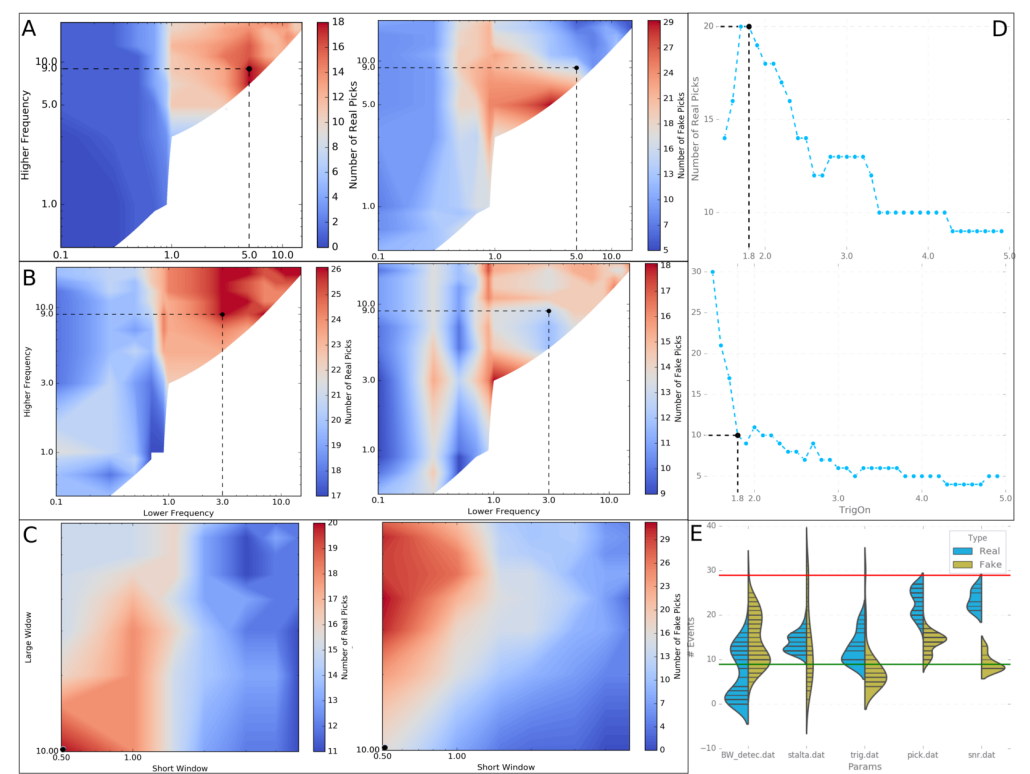

Figure 3.9: Parameter optimization results for station DUB01 - Real and false picks results for A: Butterworth of the detector, B: Butterworth of the picker, C: STA/LTA of the detector, D: TrigOn of the detector. E: Probability density function for each parameter results, Blue and yellow bars with the distributions for real and false picks results respectively. The green line is the number of automatic picks before optimization, and the red line is the number of manual picks. 


\section{ADDITIONAL RESULTS}

From figure 3.9 we can conclude:

1. The most critical parameters to be optimized are the Butterworth of the detector and the Butterworth of the picker. The first increases the number of picks from 9 to 18 , and the second increases from 20 to 27 picks.

2. The detector's Butterworth has a single maximum of real picks matching to $B W(4,5.0,9.0)$. The Butterworth of the detector has several local maxima. These solutions have a similar number of real picks, but they increase the number of false picks, and therefore the $B W(4,3.0,9.0)$ solution was chosen.

3. In figure $3.9 \mathrm{C}$, there is only one solution with the maximum number of real picks and a low number of false picks.

4. Figure 3.9 $\mathrm{D}$ shows the importance of optimizing the Trigon. It is essential to reduce the number of false picks.

5. Figure 3.9. E shows that optimizing the Bw_detect exceeding the number of automatic picks generated before this work (green line), and optimizing the Bw_pick increases the number of real picks up to $100 \%$ of the manual picks (red line).

\subsubsection{Numerous false picks}

Stations, with poor results for false picks regardless of their real picks' performance.

Stations with false picks higher than the $50 \%$ of manual picks ALF01, SJMB, BB19B, IPMB

Following, we present graphs showing the result after parameter optimization for the stations ALF01 and SJMB. These stations were chosen to represent all stations from this group: 


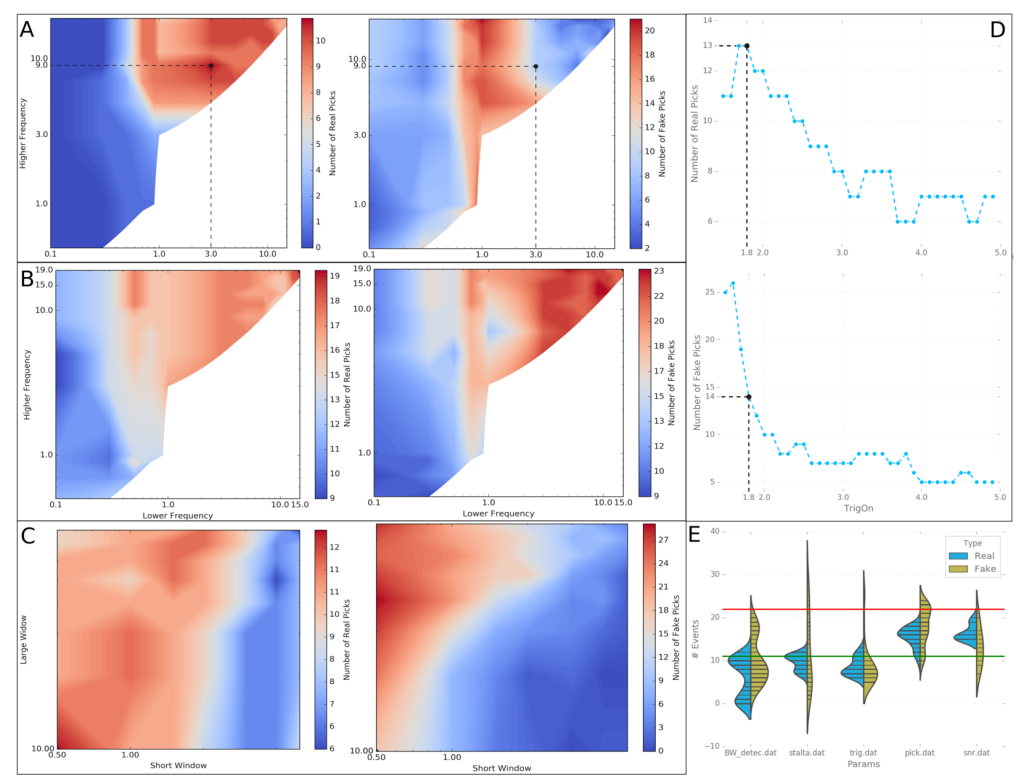

Figure 3.10: Parameter optimization results for station ALF01 - Real and false picks results for A: Butterworth of the detector, B: Butterworth of the picker, C: STA/LTA of the detector, D: TrigOn of the detector. E: Probability density function for each parameter results, Blue and yellow bars with the distributions for real and false picks results respectively. The green line is the number of automatic picks before optimization, and the red line is the number of manual picks.

From figure 3.10 we can conclude:

1. From figure 3.10 -B, the value chosen for the Butterworth of the picker is $B W(4,15,19)$, we can conclude that although the real picks increase from 13 to 20, the false picks also increase up to 21 . False picks decrease for frequency values below $3 \mathrm{~Hz}$. There is a good solution close to $B W(4,1.0,8.0)$ where there is a significant decrease in false picks, keeping numerous real picks. The above shows the necessity to change the objective value from recall to f1-score.

2. Figure 3.10 - D show that increasing the trigOn value above the chosen value of 1.8 , the number of false picks and real picks decreases; thus, this is not an adequate solution. 


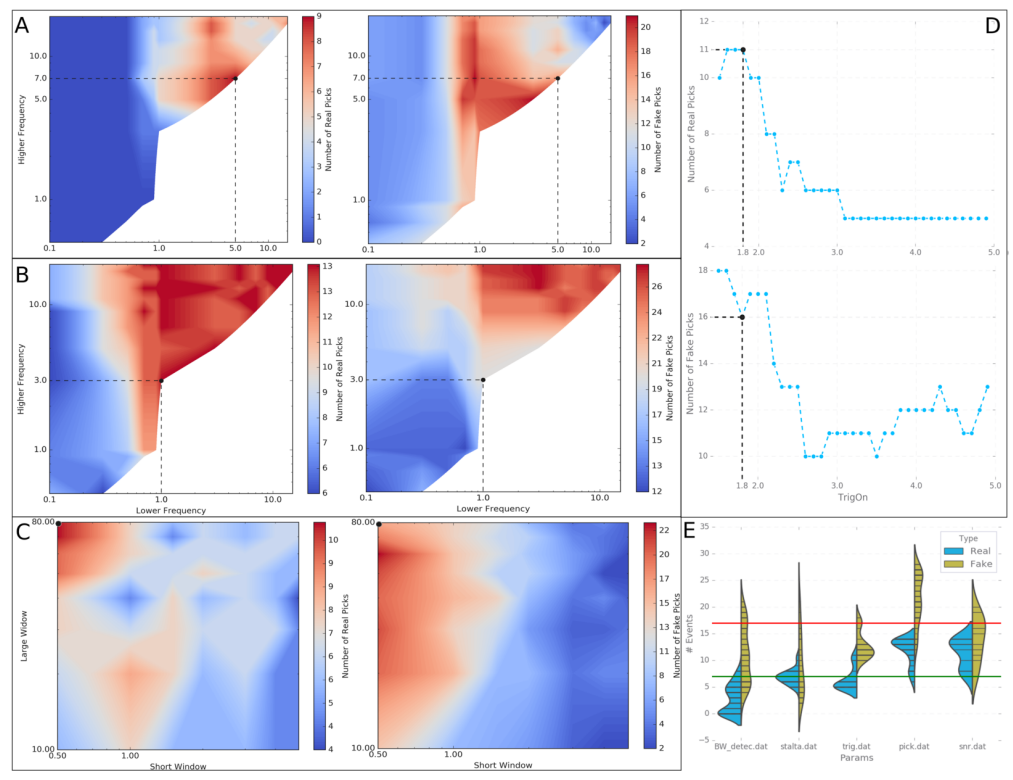

Figure 3.11: Parameter optimization results for station SJMB. - Real and false picks results for A: Butterworth of the detector, B: Butterworth of the picker, C: STA/LTA of the detector, D: TrigOn of the detector. E: Probability density function for each parameter results, Blue and yellow bars with the distributions for real and false picks results respectively. The green line is the number of automatic picks before optimization, and the red line is the number of manual picks.

From figure 3.11 we can conclude:

1. In figure 3.11 C 80 seconds LTA value is shown, we assume that this anomalous value produces numerous false events. On the other hand, by analyzing results for other parameters, we can conclude that the number of false picks results from all optimization processes. In figure 3.11 - C, there is a solution with $S T A L T A(1.20)$ with enough real picks and a decrease in false picks up to 14 .

2. Figure 3.11 A shows high-frequency solutions such as $B W(4,14,18)$ that keep a good number of real picks simultaneously with a low number of false picks. Using f1-score instead of recall solutions with fewer false picks could be selected.

3. The overall behavior of this station presents a high number of false picks. Therefore, the station must be quite noisy; thus, seismic noise could be another essential factor in the optimization process. 


\subsubsection{Good performance on false picks}

Stations, with good results for false picks regardless of their real picks' performance.

Stations with 1 or 0 false picks: RIB01, PET01, SALV, TQJ4A, SNDB, CRSM, TQJ2B

Stations with less than six manual picks were excluded.

Following, we present graphs showing the result after parameter optimization for the stations RIB01 and SALV. These stations were chosen to represent all stations from this group:

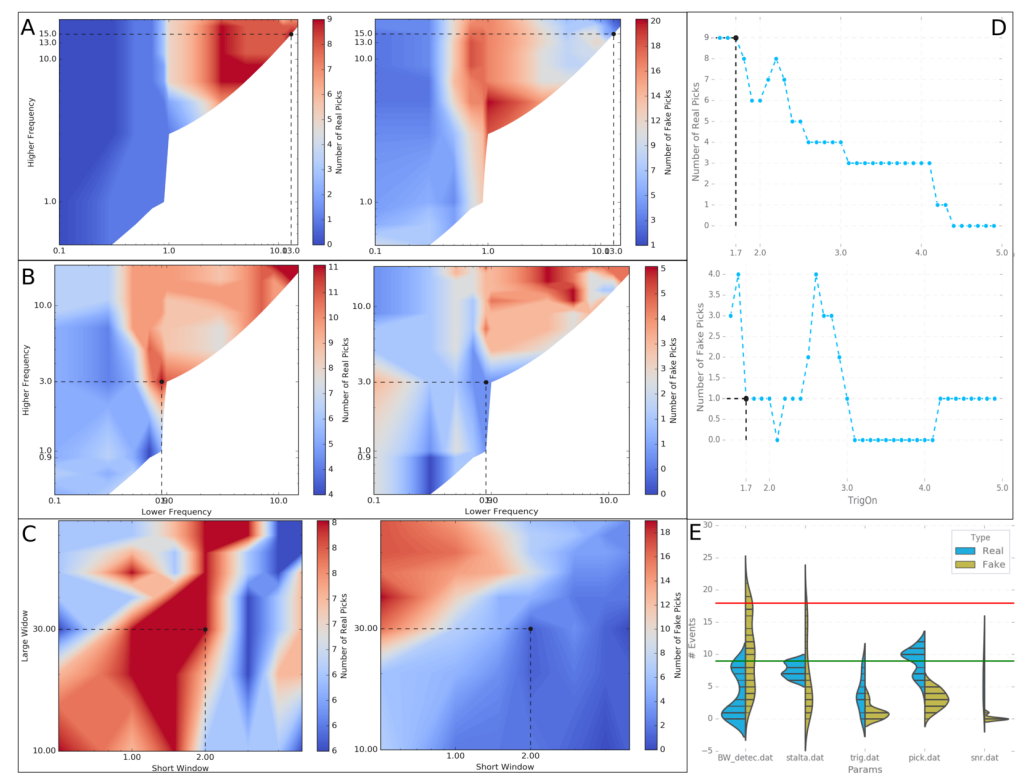

Figure 3.12: Parameter optimization results for station RIB01 - Real and false picks results for A: Butterworth of the detector, B: Butterworth of the picker, C: STA/LTA of the detector, D: TrigOn of the detector. E: Probability density function for each parameter results, Blue and yellow bars with the distributions for real and false picks results respectively. The green line is the number of automatic picks before optimization, and the red line is the number of manual picks.

From figure 3.12 we can conclude:

1. Figure 3.12 - A shows that using high-frequency filter $B W(4.13 .15)$ produced the maximum number of real picks and only 1 false pick. High-frequency detector Butterworth was the selected solution for several stations such as RIB01, PET01, 


\section{ADDITIONAL RESULTS}

and TQJ4A (See table 3.3). For these stations, a low number of false picks was produced.

2. Figure 3.12 -B shows several solutions with good results from real picks, although few of them have a low number of false picks. The selected solution $B W(4,0.9,3.0)$ has excellent performance for real and fake picks.

3. Using high-frequency detector Butterworth solutions and a low-frequency picker Butterworth selected for RIB01, PET01, and TQj4A stations produced a low number of false picks.

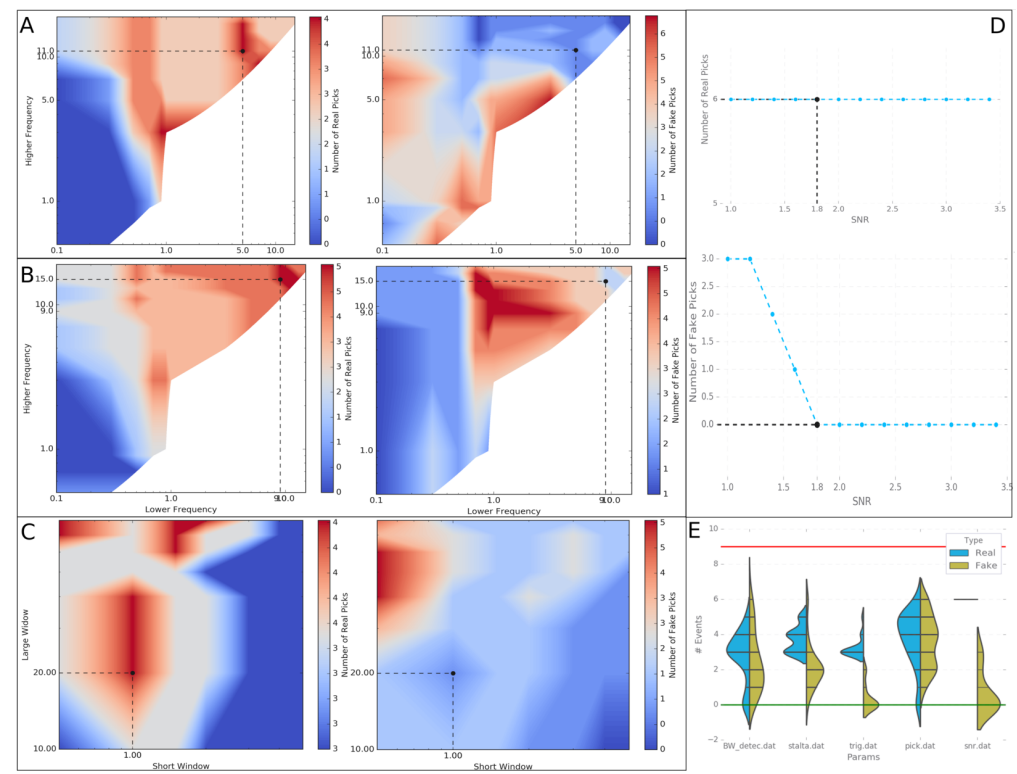

Figure 3.13: Parameter optimization results for station SALV - Real and false picks results for A: Butterworth of the detector, B: Butterworth of the picker, C: STA/LTA of the detector, D: TrigOn of the detector. E: Probability density function for each parameter results, Blue and yellow bars with the distributions for real and false picks results respectively. The green line is the number of automatic picks before optimization, and the red line is the number of manual picks.

From figure 3.13 we can conclude:

1. Figures with results of detector TrigOn presented for the other stations were replaced for the signal-to-noise ratio (SNR) of the picker (figure 3.13-D). The above due to the anomalous SNR value of 1.8 selected for this station. 
2. Figure 5-D show that the number of real picks does not decrease when SNR increases; therefore, a higher SNR value than usually can be selected. Value of 1.8 results in zero false picks.

\subsubsection{Few manual picks}

The stations highlighted in pink at the bottom of table 3.3 have less than five manual picks. These stations have been omitted from the analysis, and their results are ignored. The methodology used is very sensitive to the quantity and quality of manual picks; therefore, it is evident the necessity to select events from a more extended period to increase the number of manual picks considerably.

\subsubsection{Few real automatic picks before optimization process}

Stations, with $5 \%$ or less automatic picks before optimization over manual picks.

PARB, PMNB, PET01, VABB, SALV, TQJ4A, TQJ2B, PP1B

Real picks after the optimization process increased considerably for these stations. PMNB and TQJ2B stations, improving from $0 \%$ to $80 \%$ and from $0 \%$ to $100 \%$, respectively. 


\section{ADDITIONAL RESULTS}

\begin{tabular}{|c|c|c|c|c|c|c|c|c|c|c|c|c|}
\hline & \multicolumn{2}{|c|}{ BW Detector } & \multicolumn{2}{|c|}{ STALTA } & \multicolumn{3}{|c|}{ BW Picker } & \multirow{2}{*}{$\frac{\text { SNR }}{\min }$} & \multicolumn{4}{|c|}{ Picks } \\
\hline STA & lf & $\mathrm{hf}$ & STA & LTA & trigOn & lf & hf & & $\operatorname{man}$ & auto & real & false \\
\hline DUB01 & 5.0 & 9.0 & 0.5 & 10 & 1.8 & 3.0 & 9.0 & 1.0 & 29 & $31 \%$ & $93 \%$ & 13 \\
\hline VAS01 & 9.0 & 19.0 & 1.0 & 20 & 1.7 & 0.9 & 7.0 & 1.0 & 26 & $38 \%$ & $73 \%$ & 8 \\
\hline ALF01 & 3.0 & 9.0 & 0.5 & 10 & 1.8 & 15.0 & 19.0 & 1.0 & 22 & $50 \%$ & $90 \%$ & 21 \\
\hline PARB & 5.0 & 11.0 & 1.0 & 10 & 1.6 & 3.0 & 13.0 & 1.0 & 21 & $5 \%$ & $62 \%$ & 5 \\
\hline DIAM & 0.7 & 13.0 & 1.5 & 40 & 1.7 & 0.5 & 7.0 & 1.4 & 18 & $39 \%$ & $78 \%$ & 6 \\
\hline RIB01 & 13.0 & 15.0 & 2.0 & 30 & 1.7 & 0.9 & 3.0 & 1.0 & 18 & $50 \%$ & $67 \%$ & 1 \\
\hline BSCB & 5.0 & 11.0 & 1.0 & 10 & 1.7 & 0.7 & 2.0 & 1.0 & 18 & $44 \%$ & $78 \%$ & 4 \\
\hline SJMB & 5.0 & 7.0 & 0.5 & 80 & 1.8 & 1.0 & 3.0 & 1.4 & 17 & $41 \%$ & $82 \%$ & 17 \\
\hline PMNB & 7.0 & 17.0 & 0.5 & 20 & 1.7 & 1.0 & 3.0 & 1.0 & 15 & $0 \%$ & $80 \%$ & 5 \\
\hline РET01 & 13.0 & 15.0 & 1.0 & 20 & 1.7 & 0.3 & 5.0 & 1.0 & 13 & $0 \%$ & $54 \%$ & 1 \\
\hline VABB & 9.0 & 19.0 & 1.0 & 20 & 1.7 & 3.0 & 13.0 & 1.2 & 13 & $0 \%$ & $61 \%$ & 5 \\
\hline GUA01 & 3.0 & 11.0 & 2.0 & 20 & 1.7 & 0.9 & 9.0 & 1.0 & 12 & $33 \%$ & $92 \%$ & 3 \\
\hline BB19B & 7.0 & 9.0 & 1.0 & 20 & 1.7 & 3.0 & 7.0 & 1.0 & 10 & $10 \%$ & $60 \%$ & 6 \\
\hline JANB & 13.0 & 15.0 & 1.0 & 20 & 1.7 & 0.9 & 19.0 & 1.2 & 10 & $40 \%$ & $90 \%$ & 3 \\
\hline SALV & 5.0 & 11.0 & 1.0 & 20 & 1.6 & 9.0 & 15.0 & 1.8 & 9 & $0 \%$ & $67 \%$ & 0 \\
\hline IPMB & 1.0 & 13.0 & 0.5 & 30 & 1.7 & 0.9 & 5.0 & 1.2 & 9 & $11 \%$ & $78 \%$ & 6 \\
\hline TQJ4A & 11.0 & 13.0 & 1.0 & 20 & 1.6 & 0.1 & 0.7 & 3.4 & 9 & $0 \%$ & $44 \%$ & 1 \\
\hline AQDB & 5.0 & 7.0 & 1.0 & 20 & 1.7 & 15.0 & 19.0 & 1.0 & 8 & $12 \%$ & $87 \%$ & 2 \\
\hline ARAG & 5.0 & 17.0 & 1.0 & 20 & 1.7 & 0.9 & 7.0 & 1.0 & 7 & $14 \%$ & $57 \%$ & 2 \\
\hline SNDB & 1.0 & 17.0 & 1.0 & 20 & 1.7 & 3.0 & 5.0 & 2.6 & 7 & $28 \%$ & $57 \%$ & 0 \\
\hline CRSM & 3.0 & 13.0 & 1.0 & 20 & 1.7 & 0.5 & 0.9 & 1.0 & 7 & $71 \%$ & $100 \%$ & 0 \\
\hline TQJ2B & 9.0 & 17.0 & 1.0 & 10 & 1.6 & 0.3 & 3.0 & 1.0 & 7 & $0 \%$ & $100 \%$ & 0 \\
\hline PP1B & 5.0 & 11.0 & 1.0 & 20 & 1.6 & 0.3 & 5.0 & 1.0 & 6 & $0 \%$ & $67 \%$ & 2 \\
\hline AMBA & 5.0 & 13.0 & 1.0 & 10 & 1.6 & 0.5 & 9.0 & 1.0 & 6 & $16 \%$ & $50 \%$ & 1 \\
\hline FRTB & 7.0 & 9.0 & 1.0 & 10 & 1.6 & 13.0 & 19.0 & 1.0 & 6 & $16 \%$ & $83 \%$ & 1 \\
\hline ALGR & 3.0 & 9.0 & 3.0 & 10 & 1.7 & 0.3 & 0.5 & 1.0 & 5 & $40 \%$ & $60 \%$ & 0 \\
\hline PCMB & 1.0 & 9.0 & 1.0 & 30 & 1.7 & 0.7 & 3.0 & 1.2 & 5 & $20 \%$ & $80 \%$ & 0 \\
\hline PDRB & 3.0 & 5.0 & 1.0 & 20 & 1.7 & 0.5 & 15.0 & 1.0 & 4 & $0 \%$ & $75 \%$ & 1 \\
\hline MURT & 0.9 & 19.0 & 2.0 & 20 & 1.7 & 3.0 & 5.0 & 1.0 & 4 & $25 \%$ & $100 \%$ & 0 \\
\hline ABR01 & 9.0 & 11.0 & 0.5 & 10 & 1.7 & 0.7 & 5.0 & 2.0 & 4 & $25 \%$ & $75 \%$ & 0 \\
\hline AZCA & 1.0 & 7.0 & 1.0 & 10 & 1.7 & 0.1 & 13.0 & 1.0 & 4 & $50 \%$ & $100 \%$ & 0 \\
\hline ITAB & 5.0 & 19.0 & 1.0 & 10 & 1.6 & 0.1 & 0.5 & 1.0 & 4 & $50 \%$ & $100 \%$ & 0 \\
\hline VILB & 3.0 & 13.0 & 1.0 & 20 & 1.5 & 0.1 & 0.5 & 1.0 & 3 & $0 \%$ & $67 \%$ & 0 \\
\hline TIJ01 & 0.9 & 5.0 & 1.0 & 20 & 1.7 & 0.1 & 0.9 & 1.0 & 3 & $0 \%$ & $33 \%$ & 0 \\
\hline $\mathrm{C} 2 \mathrm{SB}$ & 3.0 & 17.0 & 1.0 & 10 & 1.6 & 0.1 & 3.0 & 1.0 & 3 & $0 \%$ & $33 \%$ & 0 \\
\hline CNLB & 3.0 & 11.0 & 1.0 & 20 & 4.1 & 0.5 & 7.0 & 1.0 & 3 & $33 \%$ & $66 \%$ & 0 \\
\hline ITQB & 3.0 & 9.0 & 1.0 & 10 & 1.6 & 0.1 & 7.0 & 1.0 & 3 & $33 \%$ & $100 \%$ & 0 \\
\hline BDQN & 0.1 & 11.0 & 1.0 & 20 & 1.6 & 0.1 & 9.0 & 1.0 & 3 & $0 \%$ & $66 \%$ & 1 \\
\hline LDASE & 0.9 & 7.0 & 1.0 & 10 & 1.6 & 0.1 & 17.0 & 1.0 & 3 & $67 \%$ & $100 \%$ & 0 \\
\hline
\end{tabular}

Table 3.3: Table with the results of the parameters optimized. BW: Butterworth. If: low frequency. hf: high frequency. man: manual picks. auto: percentage of manual picks identified automatically before optimization. real: percentage of manual picks identified automatically after optimization. fake: false automatic picks after optimization. Blue color: stations with more than $75 \%$ of manual picks identified automatically after optimization. Red color: stations with poor results of false picks, stations with less than $5 \%$ of manual picks identified before optimization, and parameters identified which generate poor results of real or false picks. Pink color: stations with less than five manual picks. 


\section{4}

\section{Conclusion}

\subsection{Final Conclusion}

The need to improve the performance of the automatic processing system in seismological centers was the principal problem to solve during this study.

Detector STA/LTA and picker AIC algorithms were chosen as the principal part for being the first steps of the automatic processing.

Two possible solutions were planned at the beginning, one was an intensive analysis of seismic noise and the other was that using manual picks as training data it is possible to train a set of stations to reproduce these data automatically. The second approach was chosen for the possibility of using all onset times available on databases such us the principal input for this work. We developed a code using grid-search to maximize the recall. Three principal problems were found: 1) selected data set was not enough, 2) grid-search is very computationally expensive and 3) not only real picks must be maximized but false picks must be minimized. Thus, a bigger set of data was used, Bayesian methodology was implemented and f1-score was maximized. Finally both grid search with recall and Bayesian with f1-score produced excellent result by doubling automatic picks and automatic locations. Grid search proved to be an excellent tool to understand and analyze the system performance while Bayesian optimization has better efficiency. Using recall as score to maximize produced the higher number of real automatic picks but also of false picks. On the other hand, using f1-score produce high number of real picks and low number of false picks. 


\section{CONCLUSION}

\subsection{Principal Achievements}

First, detection and picker parameters for 52 stations of the RSBR were optimized, which are essential to increase the number of automatic picks and therefore automatic locations. Second, cooperation with seismologist from RSNC was fundamental to improve the methodology and to prove our method using stations from both networks. A new methodology is proposed in this study and we hope that this could be used for other seismological centers to improve their results.

Other achievements are the paper attached on chapter two of this document and submitted to the "Bulletin of the Seismological Society of America" and abstract submitted to "Seismological Society 2021 Annual Meeting" and "4th Simpósio Brasileiro de Sismologia" part of the "17th International Congress of the Brazilian Geophysical Society".

\subsection{Future Work}

In this study, automatic locations were calculated using the resulting picks after the optimization process. Parameters used in grouping picks and location algorithms were not optimized; therefore, location results can still improve. Parameter optimization for other algorithms used during automatic processing, such as grouping picks, locator, or origin associator, is necessary to get the most out of the system.

Going further, optimization could be applied to a specific training data to increase even more the automatic picks. To do that, manual events could be grouped by magnitude ranges and location to perform an independently optimization processes for each group of events. 


\section{Bibliography}

Akaike, H. (1998). Information Theory and an Extension of the Maximum Likelihood Principle, pages 199-213. Springer New York, New York, NY. 5

Allen, R. (1982). Automatic phase pickers: Their present use and future prospects. Bulletin of the Seismological Society of America, 72(6B):S225-S242. 3,4

Allen, R. V. (1978). Automatic earthquake recognition and timing from single traces. Bulletin of the Seismological Society of America, 68(5):1521-1532. 3

Baer, M. and Kradolfer, U. (1987). An automatic phase picker for local and teleseismic events. Bulletin of the Seismological Society of America, 77(4):1437-1445. 3

Bergstra, J., Bardenet, R., Bengio, Y., and Kégl, B. (2011a). Algorithms for hyperparameter optimization. In Proceedings of the 24th International Conference on Neural Information Processing Systems, NIPS'11, page 2546-2554, Red Hook, NY, USA. Curran Associates Inc. 11

Bergstra, J. S., Bardenet, R., Bengio, Y., and Kégl, B. (2011b). Algorithms for hyperparameter optimization. In Shawe-Taylor, J., Zemel, R. S., Bartlett, P. L., Pereira, F., and Weinberger, K. Q., editors, Advances in Neural Information Processing Systems 24, pages 2546-2554. Curran Associates, Inc. 8, 9

Betrò, B. (1992). Bayesian methods in global optimization. In Gritzmann, P., Hettich, R., Horst, R., and Sachs, E., editors, Operations Research '91, pages 16-18, Heidelberg. Physica-Verlag HD. 10

Bianchi, M. B., Assumpção, M., Rocha, M. P., Carvalho, J. M., Azevedo, P. A., Fontes, S. L., Dias, F. L., Ferreira, J. M., Nascimento, A. F., Ferreira, M. V., and Costa, 
I. S. L. (2018). The Brazilian Seismographic Network (RSBR): Improving Seismic Monitoring in Brazil. Seismological Research Letters, 89(2A):452-457. 2

Brochu, E., Cora, V., and Freitas, N. (2010). A tutorial on bayesian optimization of expensive cost functions, with application to active user modeling and hierarchical reinforcement learning. Computing Research Repository, abs/1012.2599. 11, 13

Chu, C.-K. and Mendel, J. (1994). First break refraction event picking using fuzzy logic systems. IEEE Transactions on Fuzzy Systems, 2(4):255-266. 5

Colombiano, S. G. (1993). Red sismologica nacional de colombia. 2

Gentili, S. and Michelini, A. (2006). Automatic picking of p and s phases using a neural tree. Journal of Seismology, 10:39-63. 5

Hendriyana, A., Bauer, K., Muksin, U., and Weber, M. (2018). AIC-based diffraction stacking for local earthquake locations at the Sumatran Fault (Indonesia). Geophysical Journal International, 213(2):952-962. 3

Hsu, C.-w., Chang, C.-c., and Lin, C.-J. (2003). A practical guide to support vector classification chih-wei hsu, chih-chung chang, and chih-jen lin. 10

Jones, D. (2001). A taxonomy of global optimization methods based on response surfaces. J. of Global Optimization, 21:345-383. 12

Leonard, M. (2000). Comparison of Manual and Automatic Onset Time Picking. Bulletin of the Seismological Society of America, 90(6):1384-1390. 2, 5

MAEDA, N. (1985). A method for reading and checking phase time in auto-processing system of seismic wave data. Zisin (Journal of the Seismological Society of Japan. 2nd ser.), 38(3):365-379. 5

Mockus, J., Tiesis, V., and Zilinskas, A. (2014). The application of bayesian methods for seeking the extremum. Towards Global Optimization, 2:117-129. 10

Olivieri, M., Michelini, A., and Lomax, A. (2007). New robust automatic earthquake locations for the italian region. 8 
Pechmann, J. C. $(1998,2006)$. Suggested picker parameter changes. Geophysical Research Abstracts, 9(05106). 8

Saragiotis, C., Hadjileontiadis, L., and Panas, S. (1999). A higher-order statisticsbased phase identification ofthree-component seismograms in a redundant wavelet transform domain. pages 396-399. 5

Sleeman, R. and van Eck, T. (1999). Robust automatic p-phase picking: an on-line implementation in the analysis of broadband seismogram recordings. Physics of the Earth and Planetary Interiors, 113(1):265 - 275. 5

Snoek, J., Larochelle, H., and Adams, R. P. (2012). Practical bayesian optimization of machine learning algorithms. In Proceedings of the 25th International Conference on Neural Information Processing Systems - Volume 2, NIPS'12, page 2951-2959, Red Hook, NY, USA. Curran Associates Inc. 11

Sokolova, M. and Lapalme, G. (2009). A systematic analysis of performance measures for classification tasks. Information Processing y Management, 45(4):427 - 437. 9

Taijia Xiao, Dong Ren, Shuanghui Lei, Junqiao Zhang, and Xiaobo Liu (2014). Based on grid-search and pso parameter optimization for support vector machine. In Proceeding of the 11th World Congress on Intelligent Control and Automation, pages $1529-1533.10$

Vassallo, M., Satriano, C., and Lomax, A. (2012). Automatic Picker Developments and Optimization: A Strategy for Improving the Performances of Automatic Phase Pickers. Seismological Research Letters, 83(3):541-554. 8

Wang, C. (2014). Optimization of svm method with rbf kernel. In Frontiers of Manufacturing and Design Science IV, volume 496 of Applied Mechanics and Materials, pages 2306-2310. Trans Tech Publications Ltd. 10

Withers, M., Aster, R., Young, C., Beiriger, J., Harris, M., Moore, S., and Trujillo, J. (1998). A comparison of select trigger algorithms for automated global seismic phase and event detection. Bulletin of the Seismological Society of America, 88:95106. 3, 7 
Wu, J., Chen, X.-Y., Zhang, H., Xiong, L.-D., Lei, H., and Deng, S.-H. (2019). Hyperparameter optimization for machine learning models based on bayesian optimizationb. Journal of Electronic Science and Technology, 17(1):26 - 40. 8, 10

Zhang, H., Thurber, C., and Rowe, C. (2003). Automatic P-Wave Arrival Detection and Picking with Multiscale Wavelet Analysis for Single-Component Recordings. Bulletin of the Seismological Society of America, 93(5):1904-1912. 5, 6 